\title{
Extension of Classical Thermodynamics to Nonequilibrium Polarization
}

\author{
Li Xiang-Yuan, Zhu Quan, \\ He Fu-Cheng and Fu Ke-Xiang \\ College of Chemical Engineering, Sichuan University \\ Chengdu 610065 \\ P. R. China
}

\section{Introduction}

Thermodynamics concerns two kinds of states, the equilibrium ones (classical thermodynamics) and the nonequilibrium ones (nonequilibrium thermodynamics). The classical thermodynamics is an extremely important theory for macroscopic properties of systems in equilibrium, but it can not be fully isolated from nonequilibrium states and irreversible processes. Therefore, within the framework of classical thermodynamics, it is significant to explore a new method to solve the questions in the nonequilibrium state. Furthermore, this treatment should be helpful for getting deep comprehension and new applications of classical thermodynamics.

For an irreversible process, thermodynamics often takes the assumption of local equilibrium, which divides the whole system into a number of macroscopic subsystems. If all the subsystems stand at equilibrium or quasi-equilibrium states, the thermodynamic functions for a nonequilibrium system can be obtained by some reasonable treatments. However, the concept of local equilibrium lacks the theoretical basis and the expressions of thermodynamic functions are excessively complicated, so it is hard to be used in practice. Leontovich[1] $\left.{ }^{1}\right]$ once introduced a constrained equilibrium approach to treat nonequilibrium states within the framework of classical thermodynamics, which essentially maps a nonequilibrium state to a constrained equilibrium one by imposing an external field. In other words, the definition of thermodynamic functions in classical thermodynamics is firstly used in constrained equilibrium state, and the following step is how to extend this definition to the corresponding nonequilibrium state. This theoretical treatment is feasible in principle, but has not been paid much attention to yet. This situation is possibly resulted from the oversimplified descriptions of the Leontovich's approach in literature and the lack of practical demands. Hence on the basis of detailed analysis of additional external parameters, we derive a more general thermodynamic formula, and apply it to the case of nonequilibrium polarization. The results show that the nonequilibrium solvation energy in the continuous medium can be obtained by imposing an appropriate external electric field, which drives the nonequilibrium state to a constrained equilibrium one meanwhile keeps the charge distribution and polarization of medium fixed. 


\section{The equilibrium and nonequilibrium systems}

\subsection{Description of state}

The state of a thermodynamic system can be described by its macroscopic properties under certain ambient conditions, and these macroscopic properties are called as state parameters. The state parameters should be divided into two kinds, i.e. external and internal ones. The state parameters determined by the position of the object in the ambient are the external parameters, and those parameters, which are related to the thermal motion of the particles constituting the system, are referred to the internal parameters. Consider a simple case that the system is the gas in a vessel, and the walls of the vessel are the object in the ambient. The volume of the gas is the external parameter because it concerns only the position of the vessel walls. Meanwhile, the pressure of the gas is the internal parameter since it concerns not only the position of the vessel walls but also the thermal motion of gas molecules. All objects interacting with the system should be considered as the ambient. However, we may take some objects as one part of a new system. Therefore, the distinction between external and internal parameters is not absolute, and it depends on the partition of the system and ambient. Note that whatever the division between system and ambient, the system may do work to ambient only with the change of some external parameters.

Based on the thermodynamic equilibrium theory, the thermal homogeneous system in an equilibrium state can be determined by a set of external parameters $\left\{a_{i}\right\}$ and an internal parameter $T$, where $T$ is the temperature of the system. In an equilibrium state, there exists the caloric equation of state, $U=U\left(a_{i}, T\right)$, where $U$ is the energy of the system,system capacitysystem capacity so we can choose one of $T$ and $U$ as the internal parameter of the system. However, for a nonequilibrium state under the same external conditions, besides a set of external parameters $\left\{a_{i}\right\}$ and an internal parameter $U$ (or $T$ ), some additional internal parameters should be invoked to characterize the nonequilibrium state of an thermal homogeneous system. It should be noted that those additional internal parameters are time dependent.

\subsection{Basic equations in thermodynamic equilibrium}

In classical thermodynamics, the basic equation of thermodynamic functions is

$$
T \mathrm{~d} S=\mathrm{d} U+\sum_{i} A_{i} \mathrm{~d} a_{i}
$$

where $S, U$ and $T$ represent the entropy, energy and the temperature (Kelvin) of the equilibrium system respectively. $\left\{a_{i}\right\}$ stand for a set of external parameters, and $A_{i}$ is a generalized force which conjugates with $a_{i}$. The above equation shows that the entropy of the system is a function of a set of external parameters $\left\{a_{i}\right\}$ and an internal parameter $U$, which are just the state parameters that can be used to describe a thermal homogeneous system in an equilibrium state. So the above equation can merely be integrated along a quasistatic path. Actually, $T \mathrm{~d} S$ is the heat $\delta Q_{r}$ absorbed by the system in the infinitesimal change along a quasistatic path. $\mathrm{d} U$ is the energy and $A_{i} \mathrm{~d} a_{i}$ is the element work done by the system when external parameter $a_{i}$ changes.

It should be noticed that the positions of any pair of $A_{i}$ and $a_{i}$ can interconvert through Legendre transformation. We consider a system in which the gas is enclosed in a cylinder 
with constant temperature, there will be only one external parameter, i.e. the gas volume $V$. The corresponding generalized force is the gas pressure $p$, so eq. (2.1) can be simplified as

$$
T \mathrm{~d} S=\mathrm{d} U+p \mathrm{~d} V
$$

It shows that $S=S(U, V)$. If we define $H=U+p V$, eq. (2.2) may be rewritten as

$$
T \mathrm{~d} S=\mathrm{d}(U+p V)-V \mathrm{~d} p=\mathrm{d} H-V \mathrm{~d} p
$$

Thus we have $S=S(H, p)$. If we choose the gas pressure $p$ as the external parameter, then $V$ should be the conjugated generalized force, and the negative sign in eq. (2.3) implies that the work done by the system is positive as the pressure decreases. Furthermore, the energy $U$ in eq. (2.2) has been changed with the relation of $U+p V=H$ in eq. (2.3), in which $H$ stands for the enthalpy of the gas.

\subsection{Nonequilibrium state and constrained equilibrium state}

It is a difficult task to efficiently extend the thermodynamic functions defined in the classical thermodynamics to the nonequilibrium state. At present, one feasible way is the method proposed by Leontovich. The key of Leontovich's approach is to transform the nonequilibrium state to a constrained equilibrium one by imposing some additional external fields. Although the constrained equilibrium state is different from the nonequilibrium state, it retains the significant features of the nonequilibrium state. In other words, the constraint only freezes the time-dependent internal parameters of the nonequilibrium state, without doing any damage to the system. So the constrained equilibrium becomes the nonequilibrium state immediately after the additional external fields are removed quickly. The introduction and removal of the additional external fields should be extremely fast so that the characteristic parameters of the system have no time to vary, which provides a way to obtain the thermodynamic functions of nonequilibrium state from that of the constrained equilibrium state.

\subsection{Extension of classical thermodynamics}

Based on the relation between the constrained equilibrium state and the nonequilibrium one, the general idea of extending classical thermodynamics to nonequilibrium systems can be summarized as follows:

1. By imposing suitable external fields, the nonequilibrium state of a system can be transformed into a constrained equilibrium state so as to freeze the time-dependent internal parameters of the nonequilibrium state.

2. The change of a thermodynamic function between a constrained equilibrium state and another equilibrium (or constrained equilibrium) state can be calculated simply by means of classical thermodynamics.

3. The additional external fields can be suddenly removed without friction from the constrained equilibrium system so as to recover the true nonequilibrium state, which will further relax irreversibly to the eventual equilibrium state. Leontovich defined the entropy of the nonequilibrium state by the constrained equilibrium. In other words, entropy of the constrained equilibrium and that of the nonequilibrium exactly after the fast removal of the external field should be thought the same. 
According to the approach mentioned above, we may perform thermodynamic calculations involving nonequilibrium states within the framework of classical thermodynamics.

\section{Entropy and free energy of nonequilibrium state}

\subsection{Energy of nonequilibrium states}

For the clarity, only thermal homogeneous systems are considered. The conclusions drawn from the thermal homogeneous systems can be extended to thermal inhomogeneous ones as long as they consist of finite isothermal parts ${ }^{[1]}$. As a thermal homogeneous system is in a constrained equilibrium state, the external parameters of the system should be divided into three kinds. The first kind includes those original external parameters $\left\{a_{i}\right\}$, and they have the conjugate generalized forces $\left\{A_{i}\right\}$. The second kind includes the additional external parameters $\left\{x_{k}\right\}$, which are totally different from the original ones. Correspondingly, the generalized forces $\left\{\xi_{k}\right\}$ conjugate with $\left\{x_{k}\right\}$, where $\xi_{k}$ is the internal parameter originating from the nonequilibrium state. The third kind is a new set of external parameters $\left\{a_{l}{ }^{\prime}\right\}$, which relate to some of the original external fields and the additional external parameters, i.e.,

$$
a_{l}{ }^{\prime}=a_{l}+x_{l}{ }^{\prime}
$$

where $a_{l}$ and $x_{l}{ }^{\prime}$ stand for the original external parameter and the additional external parameter, respectively. Supposing a generalized force $A_{l}{ }^{\prime}$ conjugates with the external parameter $a_{l}{ }^{\prime}$, the basic thermodynamic equation for a constrained equilibrium state can be expressed by considering all the three kinds of external parameters, $\left\{a_{i}\right\},\left\{x_{i}\right\}$, and $\left\{a_{l}{ }^{\prime}\right\}$, i.e.

$$
T \mathrm{~d} S^{*}=\mathrm{d} U^{*}+\sum_{i} A_{i} \mathrm{~d} a_{i}+\sum_{k} \xi_{k} \mathrm{~d} x_{k}+\sum_{l} A_{l}{ }^{\prime} \mathrm{d} a_{l}{ }^{\prime}
$$

where $S^{*}$ and $U^{*}$ stand for entropy and energy of the constrained nonequilibrium state, respectively, and other terms are the work done by the system due to the changes of three kinds of external parameters. Because the introduction and removal of additional external fields are so fast that the internal parameters $\xi_{k}$ and $A_{l}{ }^{\prime}$ may remain invariant. The transformation from the constrained equilibrium state to the nonequilibrium state can be regarded adiabatic.

Beginning with this constrained equilibrium, a fast removal of the constraining forces $\left\{x_{k}\right\}$ from the system then yields the true nonequilibrium state. By this very construction, the constrained equilibrium and the nonequilibrium have the same internal variables. In particular, the nonequilibrium entropy $S^{\text {non }}$ is equal to that of the constrained equilibrium ${ }^{[1]}$

$$
S^{\text {non }}=S^{*}
$$

The energy change of the system in the fast (adiabatic) process is given as follows

$$
\Delta U=U^{\text {non }}-U^{*}=-W
$$

where $U^{\text {non }}$ denotes the energies of the true nonequilibrium, and $W$ is the work done by the system during the non-quasistatic removal of the constraining forces, i.e., 


$$
U^{\text {non }}-U^{*}=-W=-\sum_{k} \xi_{k} \int_{x_{k}}^{0} \mathrm{~d} x_{k}-\sum_{l} A_{l}{ }^{\prime} \int_{a_{l}+x_{l}}^{a_{l}} \mathrm{~d} a_{l}{ }^{\prime}=\sum_{k} \xi_{k} x_{k}+\sum_{l} A_{l}{ }^{\prime} x_{l}{ }^{\prime}
$$

where $\sum_{k} \xi_{k} x_{k}$ and $\sum_{l} A_{l}{ }^{\prime} x_{l}{ }^{\prime}$ are work done by getting rid of the second and the third kinds of additional external fields quickly. Eq. (3.5) is just the relation between the energy of the nonequilibrium state and that of the constrained equilibrium state. If $A_{l}{ }^{\prime}=0$, eq. (3.5) is reduced to the Leontovich form, i.e., (Eq.3.5 of ref 1 )

$$
U=U^{*}+\sum_{k} \xi_{k} x_{k}
$$

$A_{l}{ }^{\prime}=0$ indicates that the constraining forces $\left\{\xi_{k}\right\}$ are new internal parameters which do not exist in the original constrained equilibrium state. This means that eq. (3.5) is an extension of Leontovich's form of eq. (3.6).

If one notes that $\xi_{k}$ and $A_{k}{ }^{\prime}$ remains invariant during the fast removal of their conjugate parameters, the energy change by eq. (3.5) becomes straightforward.

\subsection{Free energies of the constrained equilibrium and nonequilibrium states}

The free energy of the constrained equilibrium state $F^{*}$ is defined as

$$
F^{*}=U^{*}-T S^{*}
$$

Differentiating on the both sides of eq. (3.7) by substituting of eq. (3.2), we have

$$
\mathrm{d} F^{*}=-S^{*} \mathrm{~d} T-\sum_{i} A_{i} \mathrm{~d} a_{i}-\sum_{k} \xi_{k} \mathrm{~d} x_{k}-\sum_{l} A_{l}{ }^{\prime} \mathrm{d} a_{l}{ }^{\prime}
$$

The free energy of the nonequilibrium state $F^{\text {non }}$ is defined as

$$
F^{\text {non }}=U^{\text {non }}-T S^{\text {non }}
$$

Subtracting eq. (3.7) from eq. (3.9), with noticing eq. (3.5), we have

$$
F^{\text {non }}-F^{*}=\sum_{k} \xi_{k} x_{k}+\sum_{l} A_{l}{ }^{\prime} x_{l}{ }^{\prime}
$$

From the above equation, $F^{\text {non }}$ can be obtained from $F^{*}$.

A particularly noteworthy point should be that $A_{l}{ }^{\prime}$ and $x_{l}{ }^{\prime}$ are not a pair of conjugates, so the sum $\sum_{l} A_{l}{ }^{\prime} x_{l}{ }^{\prime}$ in eq. (3.10) does not satisfy the conditions of a state function. This leads to that the total differential of $F^{\text {non }}$ does not exist.

Adding the sum $\sum_{l} A_{l}{ }^{\prime} a_{l}$ to both sides of eq. (3.10), the total differential can be obtained as

$$
\mathrm{d}\left(F^{\mathrm{non}}+\sum_{l} A_{l}{ }^{\prime} a_{l}\right)=-S^{\mathrm{non}} \mathrm{d} T-\sum_{i} A_{i} \mathrm{~d} a_{i}+\sum_{k} x_{k} \mathrm{~d} \xi_{k}+\sum_{l} a_{l}{ }^{\prime} \mathrm{d} A_{l}{ }^{\prime}
$$

If the third kind of external parameters do not exist, i.e., $a_{l}=0$ and $x_{l}{ }^{\prime}=0$, hence $a_{l}{ }^{\prime}=0$, eq. (3.11) is identical with that given by Leontovich[1]. Eq. (3.11) shows that if there are external 
parameters of the third kind, the nonequilibrium free energy $F^{\text {non }}$ which comes from the free energy $F^{*}$ of the constrained state does not possess a total differential. This is a new conclusion. However, it will not impede that one may use eq. (3.11) to obtain $F^{\text {non }}$, because with this method one can transform the nonequilibrium state into a constrained equilibrium state, which can be called as state-to-state treatment. This treatment does not involve the state change with respect to time, so it can realize the extension of classical thermodynamics to nonequilibrium systems.

\section{Nonequilibrium polarization and solvent reorganization energy}

In the previous sections, the constrained equilibrium concept in thermodynamics, which can be adopted to account for the true nonequilibrium state, is introduced in detail. In this section, we will use this method to handle the nonequilibrium polarization in solution and consequently to achieve a new expression for the solvation free energy. In this kind of nonequilibrium states, only a portion of the solvent polarization reaches equilibrium with the solute charge distribution while the other portion can not equilibrate with the solute charge distribution. Therefore, only when the solvent polarization can be partitioned in a proper way, the constrained equilibrium state can be constructed and mapped to the true nonequilibrium state.

\subsection{Inertial and dynamic polarization of solvent}

Theoretical evaluations of solvent effects in continuum media have attracted great attentions in the last decades. In this context, explicit solvent methods that intend to account for the microscopic structure of solvent molecules are most advanced. However, such methods have not yet been mature for general purposes. Continuum models that can handle properly long range electrostatic interactions are thus far still playing the major role. Most continuum models are concerned with equilibrium solvation. Any process that takes place on a sufficiently long timescale may legitimately be thought of as equilibration with respect to solvation. Yet, many processes such as electron transfer and photoabsorption and emission in solution are intimately related to the so-called nonequilibrium solvation phenomena. The central question is how to apply continuum models to such ultra fast processes.

Starting from the equilibrium solvation state, the total solvent polarization is in equilibrium with the solute electric field. However, when the solute charge distribution experiences a sudden change, for example, electron transfer or light absorption/emission, the nonequilibrium polarization emerges. Furthermore, the portion of solvent polarization with fast response speed can adjust to reach the equilibrium with the new solute charge distribution, but the other slow portion still keeps the value as in the previous equilibrium state. Therefore, in order to correctly describe the nonequilibrium solvation state, it is important and necessary to divide the total solvent polarization in a proper way.

At present, there are mainly two kinds of partition method for the solvent polarization. The first one was proposed by Marcus ${ }^{[2]}$ in 1956, in which the solvent polarization is divided into orientational and electronic polarization. The other one, suggested by Pekar[3], considers that the solvent polarization is composed by inertial and dynamic polarization.

The first partition method of electronic and orientational polarization is established based on the relationship between the solvent polarization and the total electric field in the solutesolvent system. We consider an electron transfer (or light absorption/emission) in solution. 
Before the process, the solute-solvent system will stay in the equilibrium state " 1 ", and then the electronic transition happens and the system will reach the nonequilibrium state " 2 " in a very short time, and finally the system will arrive to the final equilibrium state " 2 ", due to the relaxation of solvent polarization. In the equilibrium states " 1 " and " 2 ", the relationship between the total electric field $\mathbf{E}$ and total polarization $\mathbf{P}$ is expressed as

$$
\mathbf{P}_{1}^{\mathrm{eq}}=\chi_{\mathrm{s}} \mathbf{E}_{1}^{\mathrm{eq}}, \mathbf{P}_{2}^{\mathrm{eq}}=\chi_{\mathrm{s}} \mathbf{E}_{2}^{\mathrm{eq}}
$$

where $\chi_{\mathrm{s}}=\frac{\left(\varepsilon_{\mathrm{s}}-1\right)}{4 \pi}$ is the static susceptibility, with $\varepsilon_{\mathrm{s}}$ being the static dielectric constants. The superscript "eq" denotes the equilibrium state. Correspondingly, the electronic polarizations in the equilibrium states " 1 " and " 2 " are written as

$$
\mathbf{P}_{1, \mathrm{op}}=\chi_{\mathrm{op}} \mathbf{E}_{1}^{\mathrm{eq}}, \mathbf{P}_{2, \mathrm{op}}=\chi_{\mathrm{op}} \mathbf{E}_{2}^{\mathrm{eq}}
$$

where the subscript "op" represents the electronic polarization and $\chi_{\mathrm{op}}=\frac{\left(\varepsilon_{\mathrm{op}}-1\right)}{4 \pi}$ the electronic susceptibility, with $\varepsilon_{\mathrm{op}}$ being the optical dielectric constant. In solution, the electronic polarization can finish adjustment very quickly, and hence it reaches equilibrium with solute charge even if the electronic transition in the solute molecule takes place. On the other hand, it is easy to express the orientational polarization as

$$
\mathbf{P}_{1, \mathrm{or}}=\mathbf{P}_{1}^{\mathrm{eq}}-\mathbf{P}_{1, \mathrm{op}}=\chi_{\mathrm{or}} \mathbf{E}_{1}^{\mathrm{eq}}, \mathbf{P}_{2, \mathrm{or}}=\mathbf{P}_{2}^{\mathrm{eq}}-\mathbf{P}_{2, \mathrm{op}}=\chi_{\mathrm{or}} \mathbf{E}_{2}^{\mathrm{eq}}
$$

with $\chi_{\mathrm{or}}=\chi_{\mathrm{s}}-\chi_{\mathrm{op}}$. Here, $\chi_{\mathrm{or}}$ stands for the orientational susceptibility and the subscript "or" the orientational polarization. This kind of polarization is mainly contributed from the low frequency motions of the solvents.

In the nonequilibrium state " 2 ", we express the total electric field strength and solvent polarization as $\mathbf{E}_{2}^{\text {non }}$ and $\mathbf{P}_{2}^{\text {non }}$ respectively, the electronic polarization can be defined as

$$
\mathbf{P}_{2, \mathrm{op}}^{\mathrm{non}}=\chi_{\mathrm{op}} \mathbf{E}_{2}^{\mathrm{non}}
$$

At this moment, the orientational polarization keeps invariant and the value in the previous equilibrium state " 1 ", thus the total polarization is written as

$$
\mathbf{P}_{2}^{\text {non }}=\mathbf{P}_{1, \text { or }}+\mathbf{P}_{2, \text { op }}^{\text {non }}
$$

The second partition method for the polarization is based on the equilibrium relationship between the dynamic polarization and electric field. Assuming that the solvent only has the optical dielectric constant $\varepsilon_{\text {op }}$, the dynamic electric field strength and the polarization in equilibrium state " 1 " and " 2 " can be expressed as

$$
\begin{gathered}
\mathbf{P}_{1, \mathrm{dy}}=\chi_{\mathrm{op}} \mathbf{E}_{1, \mathrm{dy}}, \\
\mathbf{P}_{2, \mathrm{dy}}=\chi_{\mathrm{op}} \mathbf{E}_{2, \mathrm{dy}}
\end{gathered}
$$


Then the inertial polarization in an equilibrium state is defined as

$$
\mathbf{P}_{1, \text { in }}=\mathbf{P}_{1}^{\mathrm{eq}}-\mathbf{P}_{1, \mathrm{dy}}, \mathbf{P}_{2, \text { in }}=\mathbf{P}_{2}^{\mathrm{eq}}-\mathbf{P}_{2, \mathrm{dy}}
$$

where the subscripts "dy" and "in" stand for the quantities due to the dynamic and inertial polarizations. In a nonequilibrium state, the inertial polarization will be regarded invariant, and hence the total polarization is decomposed to

$$
\mathbf{P}_{2}^{\text {non }}=\mathbf{P}_{1, \text { in }}+\mathbf{P}_{2, \text { dy }}
$$

With the dynamic-inertial partition, the nonequilibrium polarization is of the following form, i.e.,

$$
\mathbf{P}_{2}^{\text {non }}=\mathbf{P}_{1, \text { in }}+\mathbf{P}_{2, \mathrm{dy}}=\mathbf{P}_{1}^{\mathrm{eq}}-\mathbf{P}_{1, \mathrm{dy}}+\mathbf{P}_{2, \mathrm{dy}}=\chi_{\mathrm{s}} \mathbf{E}_{1}^{\mathrm{eq}}-\chi_{\mathrm{op}} \mathbf{E}_{1, \mathrm{dy}}+\chi_{\mathrm{op}} \mathbf{E}_{2, \mathrm{dy}}
$$

According to the inertial-dynamic partition, the picture of the nonequilibrium state " 2 " is very clear that the invariant part from equilibrium to nonequilibrium is the inertial polarization and the dynamic polarization responds to the solute charge change without time lag in nonequilibrium state, being equal to the dynamic polarization in equilibrium state " 2 ".

\subsection{Constrained equilibrium by external field and solvation energy in nonequilibrium state}

Based on the inertial-dynamic polarization partition, the thermodynamics method introduced in the previous sections can be adopted to obtain the solvation energy in nonequilibrium state, which is a critical problem to illustrate the ultra-fast dynamical process in the solvent.

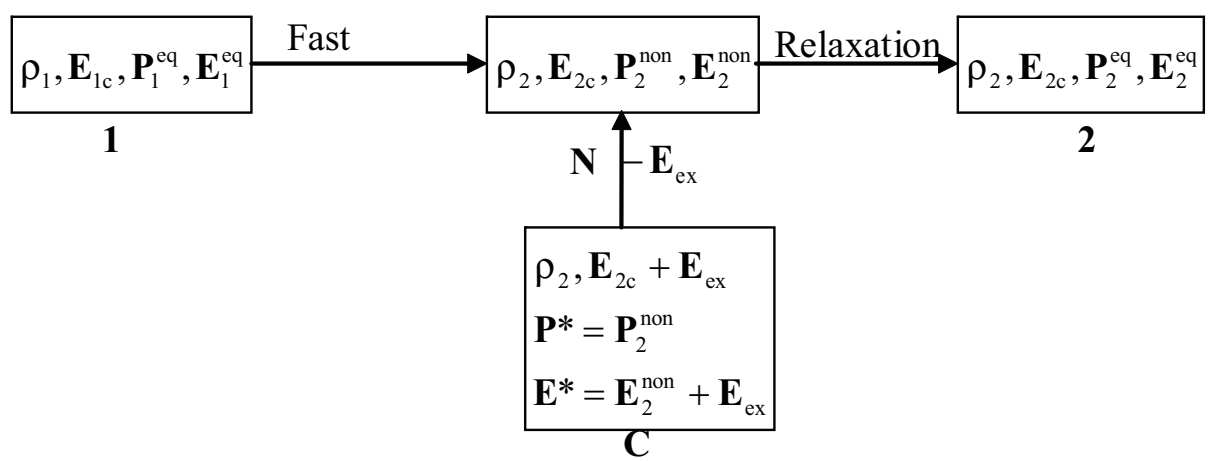

Scheme 1.

In the real solvent surroundings, the solvation energy is composed of three contributions: the cavitation energy, the dispersion-repulsion energy and electrostatic solvation energy. The cavitation energy, needed to form the solute cavity, will not change from the equilibrium " 1 " to the nonequilibrium state " 2 " due to the fixed solute structure. At the same time, the dispersion-repulsion energy is supposed invariant here. Therefore, the most important contribution to the solvation energy change from equilibrium to nonequilibrium 
is the electrostatic part, and the electrostatic solvation energy, which measures the free energy change of the medium, simplified as solvation energy in the following paragraphs, is the research focus for the ultrafast process in the medium.

As shown in Scheme 1, we adopt the letter " $N$ " to denote the nonequilibrium state, which has the same solute electric field $\mathbf{E}_{2 c}$ as equilibrium state " 2 ". The differences of polarization strength and polarization field strength between states " $\mathrm{N}$ " and " 2 " in scheme 1 can be expressed as

$$
\begin{gathered}
\mathbf{P}^{\prime}=\mathbf{P}_{2}^{\text {non }}-\mathbf{P}_{2}^{\text {eq }}=\Delta \mathbf{P}_{\text {dy }}-\Delta \mathbf{P}_{\text {eq }}=-\Delta \mathbf{P}_{\text {in }} \\
\mathbf{E}_{\mathrm{p}}^{\prime}=\mathbf{E}_{2}^{\text {non }}-\mathbf{E}_{2}^{\text {eq }}=\Delta \mathbf{E}_{\mathrm{dy}}-\Delta \mathbf{E}_{\text {eq }}=-\Delta \mathbf{E}_{\text {in }}
\end{gathered}
$$

with

$$
\Delta \mathbf{M}_{\mathrm{k}}=\mathbf{M}_{2, \mathrm{k}}-\mathbf{M}_{1, \mathrm{k}} \quad\left(\mathrm{k}=\text { " } \mathrm{dy} \text { ", "in" or " } \mathrm{eq}^{\prime \prime}\right)
$$

where $\mathbf{M}$ can be electric filed $\mathbf{E}$ or polarization $\mathbf{P}$. In eqs. (4.11) and (4.12), $\mathbf{P}^{\prime}$ is hereafter called the residual polarization which will disappear when the polarization relaxation from state " $N$ " to the final equilibrium state " 2 " has finished after enough long time. $\mathbf{E}_{\mathrm{p}}^{\prime}$ is actually a polarization field resulted from $\mathbf{P}^{\prime}$.

In order to obtain the solvation energy for the nonequilibrium state " $\mathrm{N}$ ", we can construct a constrained equilibrium state, denoted as state " $C$ " in scheme 1, by imposing an external field $\mathbf{E}_{\text {ex }}$ from the ambient on the equilibrium state " 2 ", which produces the residual polarization $\mathbf{P}^{\prime}$ and the corresponding polarization field $\mathbf{E}_{\mathrm{p}}^{\prime}$. It is clear that $\mathbf{P}^{\prime}=\chi_{\mathrm{s}}\left(\mathbf{E}_{\mathrm{ex}}+\mathbf{E}_{\mathrm{p}}^{\prime}\right)$ in the medium with the dielectric constant $\varepsilon_{\mathrm{s}}$. Thus the total electric field $\mathrm{E}^{\prime}$ due to the external field in the medium with the dielectric constant $\varepsilon_{s}$ can be expressed as

$$
\mathbf{E}^{\prime}=\mathbf{E}_{\mathrm{ex}}+\mathbf{E}_{\mathrm{p}}^{\prime}=\frac{\mathbf{P}^{\prime}}{\chi_{\mathrm{s}}}=\frac{\Delta \mathbf{P}_{\mathrm{dy}}-\Delta \mathbf{P}_{\mathrm{eq}}}{\chi_{\mathrm{s}}}=\frac{\chi_{\mathrm{op}}}{\chi_{\mathrm{s}}} \Delta \mathbf{E}_{\mathrm{dy}}-\Delta \mathbf{E}_{\mathrm{eq}}
$$

Combining eqs. (4.12) and (4.13), the external field strength can be defined as[4-6]

$$
\mathbf{E}_{\mathrm{ex}}=\mathbf{E}^{\prime}-\mathbf{E}_{\mathrm{p}}^{\prime}=\frac{\chi_{\mathrm{op}}-\chi_{\mathrm{s}}}{\chi_{\mathrm{s}}} \Delta \mathbf{E}_{\mathrm{dy}}=\frac{\varepsilon_{\mathrm{op}}-\varepsilon_{\mathrm{s}}}{\varepsilon_{\mathrm{s}}-1} \Delta \mathbf{E}_{\mathrm{dy}}
$$

Through the introduction of the external field, the constrained equilibrium state has been constructed as

$$
\begin{aligned}
& \mathbf{E}_{\mathrm{c}}^{*}=\mathbf{E}_{2 \mathrm{c}}+\mathbf{E}_{\mathrm{ex}} \\
& \mathbf{E}^{*}=\mathbf{E}_{2}^{\text {non }}+\mathbf{E}_{e x} \\
& \mathbf{P}^{*}=\mathbf{P}_{2}^{\text {non }}=\mathbf{P}_{2}^{\text {eq }}+\mathbf{P}^{\prime}
\end{aligned}
$$

where $\mathbf{E}_{c}^{*}$ is the solute electric field in vacuum. In constrained equilibrium state, the polarization, entropy and solute charge distribution are the same as the nonequilibrium 
state " $\mathrm{N}$ ". It is shown in eq. (4.15) that nonequilibrium polarization $\mathbf{P}_{2}^{\text {non }}$ equilibrates with solute and external electric field $\mathbf{E}_{2 \mathrm{c}}+\mathbf{E}_{\mathrm{ex}}$ in the medium with static dielectric constant. Therefore, the only difference between the nonequilibrium state and constrained equilibrium state is the external field $\mathbf{E}_{\mathrm{ex}}$.

Now we can analyze the equilibrium and constrained equilibrium states from the view of thermodynamics. For clarity, we take the medium (or solvent) as the "system" but both the solute (free) charge and the source of $\mathbf{E}_{\mathrm{ex}}$ as the "ambient". This means that the thermodynamic system is defined to only contain the medium, while the free charges and the constraining field act as the external field. The exclusion of the free charges from the "thermodynamic system" guarantees coherent thermodynamic treatment.

Given the above definition on the "system", we now turn to present the free energy $F_{\text {sol }}$ of the medium. Here we use the subscript "sol" to indicate the quantities of the medium, or solvent. Let us calculate the change in $F_{\text {sol }}$ resulting from an infinitesimal change in the field which occurs at constant temperature and does not destroy the thermodynamic equilibrium of the medium. The free energy change of the medium for an equilibrium polarization is equal to the total free energy change of the solute-solvent system minus the self-energy change of the solute charge, i.e.,

$$
\delta F_{\text {sol }}=\frac{1}{4 \pi} \int \mathbf{E} \cdot \delta \mathbf{D d} V-\frac{1}{4 \pi} \int \mathbf{E}_{\mathrm{c}} \cdot \delta \mathbf{E}_{\mathrm{c}} \mathrm{d} V
$$

where $\mathbf{E}$ is the total electric field while $\mathbf{E}_{c}$ is the external field by the solute charge in the vacuum. $\mathbf{D}$ is the electric displacement with the definition of $\mathbf{D}=\mathbf{E}+4 \pi \mathbf{P}=\varepsilon \mathbf{E}$. Eq.(4.16) gives the free energy of the medium for an equilibrium polarization as

$$
\begin{aligned}
F_{\text {sol }} & =\frac{1}{8 \pi} \int\left(\mathbf{D} \cdot \mathbf{E}-\mathbf{E}_{\mathrm{c}} \cdot \mathbf{E}_{\mathrm{c}}\right) \mathrm{d} V \\
& =\frac{1}{8 \pi} \int\left(\mathbf{E} \cdot \mathbf{E}_{\mathrm{c}}-\mathbf{D} \cdot \mathbf{E}_{\mathrm{c}}\right) \mathrm{d} V+\frac{1}{8 \pi} \int\left(\mathbf{E}+\mathbf{E}_{\mathrm{c}}\right) \cdot\left(\mathbf{D}-\mathbf{E}_{\mathrm{c}}\right) \mathrm{d} V
\end{aligned}
$$

We note that

$$
\mathbf{E}=-\nabla \Phi, \mathbf{E}_{\mathrm{c}}=-\nabla \psi_{\mathrm{c}}
$$

where $\Phi$ is the total electric potential produced and $\psi_{\mathrm{c}}$ is the electric potential by the solute (free) charge in vacuum. With eq.(4.18), the last term in the second equality of eq.(4.17) becomes

$$
-\frac{1}{8 \pi} \int \nabla\left(\Phi+\psi_{\mathrm{c}}\right) \cdot\left(\mathbf{D}-\mathbf{E}_{\mathrm{c}}\right) \mathrm{d} V
$$

The volume integral (4.19) can be change to the following form by integration by parts:

$$
\frac{1}{8 \pi} \int\left(\Phi+\psi_{\mathrm{c}}\right) \nabla \cdot\left(\mathbf{D}-\mathbf{E}_{\mathrm{c}}\right) \mathrm{d} V=0
$$


Thus eq.(4.17) can be rewritten as[7,8]

$$
F_{\text {sol }}=-\frac{1}{2} \int \mathbf{P} \cdot \mathbf{E}_{\mathrm{c}} \mathrm{d} V
$$

We consider our nonequilibrium polarization case. For the solvent system in the constrained

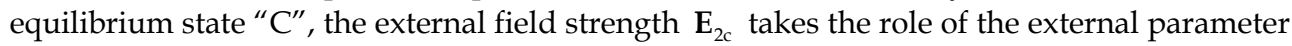
$a, \mathbf{E}_{\text {ex }}$ takes the role of $\chi^{\prime}$, and solvent polarization $\mathbf{P}^{*}=\mathbf{P}_{2}^{\text {non }}=\mathbf{P}_{2}^{\text {eq }}+\mathbf{P}^{\prime}$ takes the role of $A^{\prime}$. The total external (vacuum) electric field in this state is $\mathbf{E}_{c}^{*}=\mathbf{E}_{2 c}+\mathbf{E}_{e x}$. A constrained equilibrium can be reached through a quasistatic path, so the electrostatic free energy by an external field is of the form like eq.(4.21),

$$
F_{\text {sol }}^{*}=-\frac{1}{2} \int \mathbf{P}^{*} \cdot \mathbf{E}_{\mathrm{c}}^{*} \mathrm{~d} V=-\frac{1}{2} \int\left(\mathbf{P}_{2}^{\mathrm{eq}}+\mathbf{P}^{\prime}\right) \cdot\left(\mathbf{E}_{\mathrm{ex}}+\mathbf{E}_{2 \mathrm{c}}\right) \mathrm{d} V
$$

Similarly, the electrostatic free energy of the final equilibrium state " 2 " is given by

$$
F_{2, \mathrm{sol}}^{\mathrm{eq}}=-\frac{1}{2} \int \mathbf{P}_{2}^{\mathrm{eq}} \cdot \mathbf{E}_{2 \mathrm{c}} \mathrm{d} V
$$

Starting form the constrained equilibrium " $\mathrm{C}$ ", we prepare the nonequilibrium state " $\mathrm{N}$ " by removing the external $\mathbf{E}_{\mathrm{ex}}$ suddenly without friction. In this case, the constrained equilibrium will return to the nonequilbirium state. According to eq. (3.10), the nonequilibrium solvation energy is readily established as

$$
F_{2 . \mathrm{sol}}^{\mathrm{non}}=F_{\mathrm{sol}}^{*}+\int\left(\mathbf{P}_{2}^{\mathrm{eq}}+\mathbf{P}^{\prime}\right) \cdot \mathbf{E}_{\mathrm{ex}} \mathrm{d} V
$$

Substituting eq. (4.22) into eq. (4.24), the electrostatic solvation energy (it is just the electrostatic free energy of the medium) for the nonequilibrium state " $\mathrm{N}$ " is given by

$$
\begin{aligned}
F_{2, \text { sol }}^{\text {non }} & =-\frac{1}{2} \int\left(\mathbf{E}_{2 \mathrm{c}}+\mathbf{E}_{\mathrm{ex}}\right) \cdot\left(\mathbf{P}_{2}^{\mathrm{eq}}+\mathbf{P}^{\prime}\right) \mathrm{d} V+\int \mathbf{E}_{\mathrm{ex}} \cdot\left(\mathbf{P}_{2}^{\mathrm{eq}}+\mathbf{P}^{\prime}\right) \mathrm{d} V \\
& =\frac{1}{2} \int\left(\mathbf{E}_{\mathrm{ex}}-\mathbf{E}_{2 \mathrm{c}}\right) \cdot\left(\mathbf{P}_{2}^{\mathrm{eq}}+\mathbf{P}^{\prime}\right) \mathrm{d} V
\end{aligned}
$$

Eq. (4.25) can be further simplified as

$$
F_{2, \text { sol }}^{\text {non }}=-\frac{1}{2} \int \mathbf{E}_{2 \mathrm{c}} \cdot \mathbf{P}_{2}^{\mathrm{eq}} \mathrm{d} V+\frac{1}{2} \int \mathbf{E}_{\mathrm{ex}} \cdot \mathbf{P}^{\prime} \mathrm{d} V
$$

with the relationship of $\int \mathbf{E}_{2 c} \cdot \mathbf{P}^{\prime} \mathrm{d} V=\int \mathbf{E}_{\mathrm{ex}} \cdot \mathbf{P}_{2}^{\mathrm{eq}} \mathrm{d} V$, which is proved in Appendix A. Here, the first term on the right hand side of eq. (4.26) stands for the solvation energy of equilibrium state " 2 ", and the second term is just the solvent reorganization energy, i.e.,

$$
\lambda_{\mathrm{s}}=\frac{1}{2} \int \mathbf{E}_{\mathrm{ex}} \cdot \mathbf{P}^{\prime} \mathrm{d} V
$$


Therefore, it can be seen from eqs. (4.26) and (4.27) that the solvent reorganization energy is the energy stored in the medium from equilibrium state " 2 " to nonequilbirium state " 2 ", that is, the energy change of the medium resulted from the addition of $\mathbf{P}^{\prime}$ in the equilibrium state " 2 " by imposing the external field $\mathbf{E}_{\mathrm{ex}}$.

Combining eqs. (4.11), (4.14) and (4.27), we obtain the final form for the solvent reorganization energy as

$$
\lambda_{\mathrm{s}}=\frac{1}{2} \frac{\varepsilon_{\mathrm{s}}-\varepsilon_{\mathrm{op}}}{\varepsilon_{\mathrm{s}}-1} \int_{V} \Delta \mathbf{E}_{\mathrm{dy}} \cdot\left(\Delta \mathbf{P}_{\mathrm{eq}}-\Delta \mathbf{P}_{\mathrm{dy}}\right) \mathrm{d} V
$$

\subsection{Solvent reorganization energy and its application}

\subsubsection{Solvent reorganization energy and spectral shift}

Electron transfer reactions play an important role in chemistry and biochemistry, such as the break and repair of DNA, the function of enzyme and the breath of the life body. In Marcus' electron transfer theory, the total reorganization energy is composed of two contributions: the internal reorganization $\lambda_{\text {in }}$ due to the change of the reactant structure and the solvent reorganization energy $\lambda_{\mathrm{s}}$ due to the change of the solvent structure, i.e.

$$
\lambda=\lambda_{\text {in }}+\lambda_{\mathrm{s}}
$$

Marcus defined the solvent reorganization energy between the difference of the electrostatic solvation free energy between the nonequilibrium " 2 " and equilibrium " 2 " state, i.e. ${ }^{[9]}$

$$
\lambda_{\mathrm{s}}=F_{2, \mathrm{sol}}^{\mathrm{non}}-F_{2, \mathrm{sol}}^{\mathrm{eq}}
$$

In the above derivation, we have obtained the solvent reorganization energy in electric fieldpolarization representation as shown in eq.(4.27) and we also can derive another form of charge-potential representation as

$$
\lambda_{\mathrm{s}}=\frac{1}{2} \frac{\varepsilon_{\mathrm{s}}-\varepsilon_{\mathrm{op}}}{\varepsilon_{\mathrm{s}}-1} \oint_{S} \Delta \Phi_{\mathrm{dy}}\left(\Delta \sigma_{\mathrm{dy}}-\Delta \sigma_{\mathrm{eq}}\right) \mathrm{d} S
$$

The detailed derivation can be found in Appendix B.

For the different solute size, shape and charge distribution, we simplify the solute charge distribution as the multipole expansion located as the center of a spherical cavity. In the case of the solute monopole, we can obtain the concise form as

$$
\lambda_{\mathrm{s}}=\frac{1}{2} q_{\mathrm{ex}}\left(\Delta \varphi_{\mathrm{eq}}-\Delta \varphi_{\mathrm{dy}}\right)
$$

where $q_{\mathrm{ex}}$ is the external charge located at the center of the cavity to produce $\mathbf{P}^{\prime}$. For the point charge $q_{\mathrm{D}}$ and $q_{\mathrm{A}}$ locating at the centers of the electron donor and accepter spherical cavities, the form of solvent reorganization energy in two-sphere model is given as

$$
\lambda_{\mathrm{s}}=\frac{1}{2} q_{\mathrm{D}, \mathrm{ex}}\left(\Delta \varphi_{\mathrm{D}, \mathrm{eq}}-\Delta \varphi_{\mathrm{D}, \mathrm{dy}}\right)+\frac{1}{2} q_{\mathrm{A}, \mathrm{ex}}\left(\Delta \varphi_{\mathrm{A}, \mathrm{eq}}-\Delta \varphi_{\mathrm{A}, \mathrm{dy}}\right)
$$


where $q_{\mathrm{D}, \mathrm{ex}}$ and $q_{\mathrm{A} \text {,ex }}$ are the imposed external charge at the center of donor's and acceptor's spheres.

In the case of solute charge being point dipole moment at the center of a sphere, the solvent reorganization energy can be derived to

$$
\lambda_{\mathrm{s}}=\frac{1}{2} \boldsymbol{\mu}_{\mathrm{ex}} \cdot\left(\Delta \mathrm{E}_{\mathrm{p}, \mathrm{dy}}-\Delta \mathrm{E}_{\mathrm{p}, \mathrm{eq}}\right)
$$

where $\boldsymbol{\mu}_{\mathrm{ex}}$ is the external dipole at the sphere center and the subscript " $\mathrm{p}$ " denote the field strength produced by the polarization. The derivation for eqs. (4.32)-(4.33) is detailed in appendix $\mathrm{C}$.

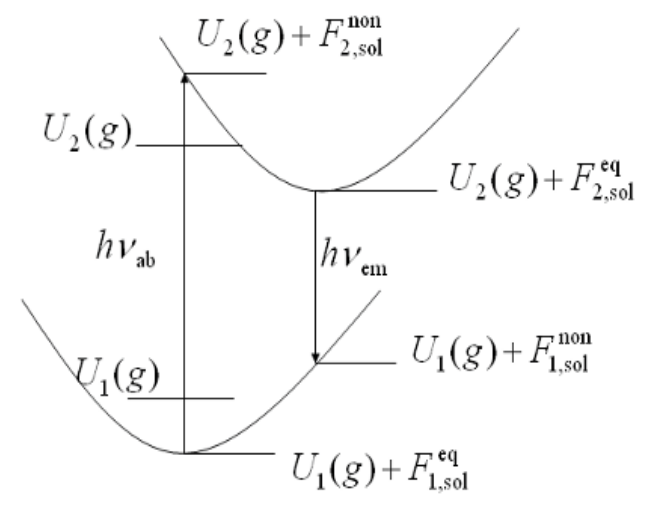

Solvation coordinate

Fig. 1. Spectral shift for the absorption and emission spectrum

Similar to the definition for the solvent reorganization energy, the spectral shifts for light absorption and emission also can be defined as shown in Figure 1. Due to the FranckCondon transition of the solute in medium, the solute-solvent system will experience the following change: starting from the equilibrium ground state, then reaching the nonequilibrium excited state, and then relaxing to the equilibrium excited state, following by the nonequilibrium ground state, and finally reaching the starting equilibrium ground state. Here we use subscripts " 1 " and " 2 " to denote the different charge distributions in ground and excited state respectively. In Figure $1, U_{i}(\mathrm{~g})(i=1,2)$ stands for the internal energies of the solute in ground state " 1 " and excited state " 2 " in vacuum. $h v_{\mathrm{ab}}$ and $h v_{\mathrm{em}}$ are the absorption and emission energy in medium respectively.

According to the traditional nonequilibrium solvation theory[2,9], the absorption spectral shift is defined as the free energy difference between nonequilibrium excited state " 2 " and equilibrium ground state " 1 ". Ignoring the self-consistence between the solute and solvent charge, the spectral shift for the absorption spectrum can be defined as the solvation energy difference between nonequilibrium excited state " 2 " and equilibrium ground state " 1 ", i.e.

$$
\Delta h v_{\mathrm{ab}}=F_{2, \mathrm{sol}}^{\mathrm{non}}-F_{1, \mathrm{sol}}^{\mathrm{eq}}
$$


Correspondingly, for the inversed process, namely, emission (or fluorescence) spectrum, the spectral shift can be expressed as

$$
\Delta h v_{\mathrm{em}}=F_{1, \mathrm{sol}}^{\mathrm{non}}-F_{2, \mathrm{sol}}^{\mathrm{eq}}
$$

According to the definitions given in eqs. (4.35) and (4.36), the positive value of $\Delta h v_{\mathrm{ab}}$ is blue shift, while the positive value of $\Delta h v_{\mathrm{em}}$ is red shift. The solvation energies for the equilibrium ground and excited states in the charge-potential presentation can be given as

$$
\begin{aligned}
& F_{1, \text { sol }}^{\mathrm{eq}}=\frac{1}{2} \int_{V} \rho_{1} \varphi_{1}^{\mathrm{eq}} \mathrm{d} V \\
& F_{2, \text { sol }}^{\mathrm{eq}}=\frac{1}{2} \int_{V} \rho_{2} \varphi_{2}^{\mathrm{eq}} \mathrm{d} V
\end{aligned}
$$

where $\varphi$ is the polarization potential and $\rho$ the charge density of the solute. According to eq. (4.26), the nonequilibrium solvation energy can be expressed in charge-potential form as

$$
\begin{aligned}
& F_{2}^{\mathrm{non}}=\frac{1}{2} \int_{V} \rho_{2} \varphi_{2}^{\mathrm{eq}} \mathrm{d} V+\lambda_{\mathrm{s}} \\
& F_{1}^{\mathrm{non}}=\frac{1}{2} \int_{V} \rho_{1} \varphi_{1}^{\mathrm{eq}} \mathrm{d} V+\lambda_{\mathrm{s}}
\end{aligned}
$$

Together with eqs. (4.35)-(4.40), the general forms for the absorption and emission spectral shift can be obtained as

$$
\begin{aligned}
& \Delta h v_{\mathrm{ab}}=\Delta F_{2}^{\mathrm{non}}-\Delta F_{1}^{\mathrm{eq}}=\lambda_{\mathrm{s}}+\frac{1}{2} \int_{V}\left(\rho_{2} \varphi_{2}^{\mathrm{eq}}-\rho_{1} \varphi_{1}^{\mathrm{eq}}\right) \mathrm{d} V \\
& \Delta h v_{\mathrm{em}}=\Delta F_{1}^{\mathrm{non}}-\Delta F_{2}^{\mathrm{eq}}=\lambda_{\mathrm{s}}-\frac{1}{2} \int_{V}\left(\rho_{2} \varphi_{2}^{\mathrm{eq}}-\rho_{1} \varphi_{1}^{\mathrm{eq}}\right) \mathrm{d} V
\end{aligned}
$$

\subsubsection{The two-sphere model for the solvent reorganization energy}

For the electron transfer reaction between the electron donor D with charges of $q_{\mathrm{D}}$ and electron acceptor A with charge of $q_{\mathrm{A}}$, the reaction process of transferring the charge of $\Delta q$ can be described by the following equation

$$
\mathrm{D}^{q_{\mathrm{D}}}+\mathrm{B}+\mathrm{A}^{q_{\mathrm{A}}} \rightarrow \mathrm{D}^{q_{\mathrm{D}}+\Delta q}+\mathrm{B}+\mathrm{A}^{q_{\mathrm{A}}-\Delta q}
$$

where B is bridge between the donor and acceptor, $q_{\mathrm{D}}+\Delta q$ and $q_{\mathrm{A}}-\Delta q$ are the charge brought by the donor and acceptor after the electron transfer reaction. Here, we assume that all the point charges $q_{\mathrm{D}}, q_{\mathrm{A}}, q_{\mathrm{D}}+\Delta q$ and $q_{\mathrm{A}}-\Delta q$ locate at the centers of the two spheres shown in Figure 2. $r_{\mathrm{D}}$ and $r_{\mathrm{A}}$ are the radii for donor and acceptor spheres respectively. The two spheres are surrounded by the solvent with $\varepsilon_{\mathrm{s}}$, and the distance between the two spherical centers is $d$, which is assumed much larger than the radius of $r_{\mathrm{D}}$ and $r_{\mathrm{A}}$. 
Similar to the treatment by Marcus [2], ignoring the image charge effect due to the surface polarization charge, the polarization potential due to charge variation $\Delta q_{\mathrm{D}}=\Delta q$ on the surface of sphere D can be expressed as

$$
Q_{\mathrm{D}, \mathrm{s}}=\Delta q\left(\frac{1}{\varepsilon_{\mathrm{s}}}-1\right), Q_{\mathrm{D}, \mathrm{dy}}=\Delta q\left(\frac{1}{\varepsilon_{\mathrm{op}}}-1\right)
$$

in the medium of $\varepsilon_{s}$ and $\varepsilon_{\mathrm{op}}$. Correspondingly the charge variation $\Delta q_{\mathrm{A}}=-\Delta q$ in sphere A will induce the polarized charge on the surface of sphere A as

$$
Q_{\mathrm{A}, \mathrm{s}}=-\Delta q\left(\frac{1}{\varepsilon_{\mathrm{s}}}-1\right), Q_{\mathrm{A}, \mathrm{dy}}=-\Delta q\left(\frac{1}{\varepsilon_{\mathrm{op}}}-1\right)
$$

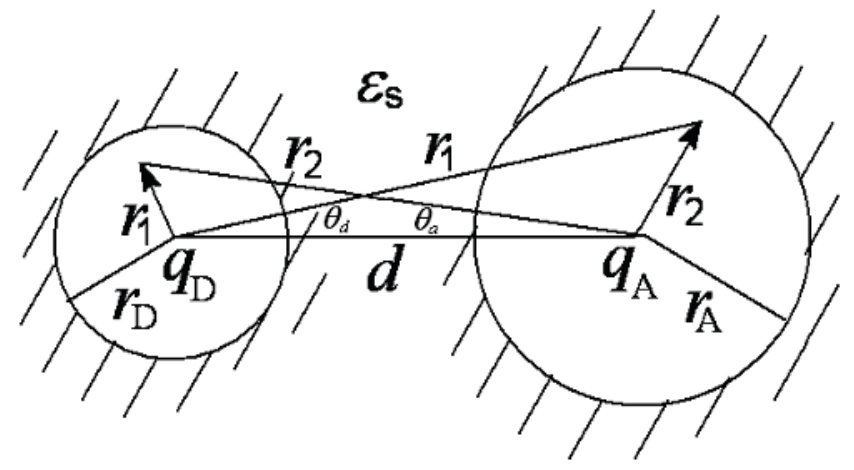

Fig. 2. Two-sphere model

Thus, in the medium of $\varepsilon_{\mathrm{op}}$, the polarization charge $Q_{D, \text { dy }}$ due to $\Delta q_{\mathrm{D}}$ will generate the polarization potential $\frac{\Delta q}{r_{D}}\left(\frac{1}{\varepsilon_{\mathrm{op}}}-1\right)$ at the center of sphere $\mathrm{D}$, and $Q_{\mathrm{A}, \mathrm{dy}}$ due to $\Delta q_{\mathrm{A}}$ will generate the polarization potential $-\frac{\Delta q}{d}\left(\frac{1}{\varepsilon_{\mathrm{op}}}-1\right)$ at the center of sphere D. Based on the principle of potential superposition, the total polarization potential at the center of sphere D can be expressed as

$$
\Delta \varphi_{\mathrm{D}, \mathrm{dy}}=\left(\frac{\Delta q}{r_{D}}-\frac{\Delta q}{d}\right)\left(\frac{1}{\varepsilon_{\mathrm{op}}}-1\right)
$$

With the similar treatment, the total polarization potential at the center of sphere A is

$$
\Delta \varphi_{\mathrm{A}, \mathrm{dy}}=-\left(\frac{\Delta q}{r_{\mathrm{A}}}-\frac{\Delta q}{d}\right)\left(\frac{1}{\varepsilon_{\mathrm{op}}}-1\right)
$$


For the solvent with dielectric constant $\varepsilon_{\mathrm{s}}$, we have

$$
\begin{gathered}
\Delta \varphi_{\mathrm{D}, \mathrm{eq}}=\left(\frac{\Delta q}{r_{D}}-\frac{\Delta q}{d}\right)\left(\frac{1}{\varepsilon_{\mathrm{s}}}-1\right) \\
\Delta \varphi_{\mathrm{A}, \mathrm{eq}}=-\left(\frac{\Delta q}{r_{\mathrm{A}}}-\frac{\Delta q}{d}\right)\left(\frac{1}{\varepsilon_{\mathrm{s}}}-1\right)
\end{gathered}
$$

With the zeroth approximation of multipole expansion for the solute charge distribution, the external charges at the position of donor and acceptor can be derived from eq. (C2) as

$$
q_{\mathrm{D}, \mathrm{ex}}=\frac{\varepsilon_{\mathrm{op}}-\varepsilon_{\mathrm{s}}}{\left(\varepsilon_{\mathrm{s}}-1\right) \varepsilon_{\mathrm{op}}} \Delta q, q_{\mathrm{A}, \mathrm{ex}}=-\frac{\varepsilon_{\mathrm{op}}-\varepsilon_{\mathrm{s}}}{\left(\varepsilon_{\mathrm{s}}-1\right) \varepsilon_{\mathrm{op}}} \Delta q
$$

Substituting eqs.(4.46)-(4.50) into eq.(4.33), the solvent reorganization energy in the twosphere and point charge model can be obtained as

$$
\lambda_{\mathrm{s}}=\frac{(\Delta q)^{2}}{2} \frac{\left(\varepsilon_{\mathrm{s}}-\varepsilon_{\mathrm{op}}\right)^{2}}{\left(\varepsilon_{\mathrm{s}}-1\right) \varepsilon_{\mathrm{s}} \varepsilon_{\mathrm{op}}^{2}}\left(\frac{1}{r_{D}}+\frac{1}{r_{A}}-\frac{2}{d}\right)
$$

It is different from the traditional Marcus result $[2,9]$

$$
\lambda_{\mathrm{M}}=\frac{(\Delta q)^{2}}{2} \frac{\left(\varepsilon_{\mathrm{s}}-\varepsilon_{\mathrm{op}}\right)}{\varepsilon_{\mathrm{s}} \varepsilon_{\mathrm{op}}}\left(\frac{1}{r_{D}}+\frac{1}{r_{A}}-\frac{2}{d}\right)
$$

The two sphere model is widely used to investigate the electron transfer reactions in solvent for its brief and simple expression. It is clear that the present two-sphere model will predict the solvent reorganization energy to be smaller than that by Marcus formula by a factor of $\frac{\varepsilon_{\mathrm{s}}-\varepsilon_{\mathrm{op}}}{\varepsilon_{\mathrm{op}}\left(\varepsilon_{\mathrm{s}}-1\right)}$.

\subsubsection{The spectral shift of photo-induced ionization energy in a single sphere}

Now we consider the simplest case for the nonequilibrium state: the solute charge distribution is point charge located at the center of the sphere with radius $a$, surrounded by the solvent with dielectric constant $\varepsilon_{\mathrm{s}}$. This model can be adopted to treat the spectral shift of the vertical ionization energy. The atomic (or ionic) photo-induced ionization process in the medium with dielectric constants $\varepsilon_{\mathrm{s}}$ or $\varepsilon_{\mathrm{op}}$ can be represented as

$$
\mathrm{B}^{Q_{1}} \stackrel{-e^{-}}{\longrightarrow} \mathrm{B}^{Q_{2}}
$$

where $Q_{2}$ and $Q_{1}$ are the solute charges before and after the ionization respectively. Induced by the charge change $\Delta Q=Q_{2}-Q_{1}$, the polarization charge on the sphere surface can be obtained as

$$
\Delta Q_{\text {eq }}^{\text {surf }}=\Delta Q\left(\frac{1-\varepsilon_{\mathrm{s}}}{\varepsilon_{\mathrm{s}}}\right)
$$




$$
\Delta Q_{\mathrm{dy}}^{\text {surf }}=\Delta Q\left(\frac{1-\varepsilon_{\mathrm{op}}}{\varepsilon_{\mathrm{op}}}\right)
$$

in the medium of $\varepsilon_{\mathrm{s}}$ and $\varepsilon_{\mathrm{op}}$, and it will generate the polarization potential at the sphere center in these two cases as

$$
\begin{gathered}
\Delta \varphi_{\mathrm{eq}}=\frac{Q_{2}-Q_{1}}{a}\left(\frac{1}{\varepsilon_{\mathrm{s}}}-1\right) \\
\Delta \varphi_{\mathrm{dy}}=\frac{Q_{2}-Q_{1}}{a}\left(\frac{1}{\varepsilon_{\mathrm{op}}}-1\right)
\end{gathered}
$$

Recalling eq. (C2), the external charge condensed at the center can be achieved as

$$
q_{\mathrm{ex}}=\frac{\varepsilon_{\mathrm{op}}-\varepsilon_{\mathrm{s}}}{\left(\varepsilon_{\mathrm{s}}-1\right) \varepsilon_{\mathrm{op}}}\left(Q_{2}-Q_{1}\right)
$$

Thus eqs. (4.39) and (4.41) can be simplified as

$$
\begin{gathered}
\lambda_{\mathrm{s}}=\frac{1}{2} q_{\mathrm{ex}}\left(\Delta \varphi_{\mathrm{eq}}-\Delta \varphi_{\mathrm{dy}}\right) \\
\Delta h \nu_{\mathrm{ab}}=\lambda_{\mathrm{s}}+\frac{1}{2}\left(Q_{2} \varphi_{2}^{\mathrm{eq}}-Q_{1} \varphi_{1}^{\mathrm{eq}}\right) \\
=\frac{1}{2} \frac{\varepsilon_{\mathrm{op}}-\varepsilon_{\mathrm{s}}}{\left(\varepsilon_{\mathrm{s}}-1\right) \varepsilon_{\mathrm{op}}}\left(Q_{2}-Q_{1}\right) \frac{Q_{2}-Q_{1}}{a}\left(\frac{1-\varepsilon_{\mathrm{s}}}{\varepsilon_{\mathrm{s}}}-\frac{1-\varepsilon_{\mathrm{op}}}{\varepsilon_{\mathrm{op}}}\right)+\frac{1}{2}\left[Q_{2} \frac{Q_{2}}{a}\left(\frac{1}{\varepsilon_{\mathrm{s}}}-1\right)-Q_{1} \frac{Q_{1}}{a}\left(\frac{1}{\varepsilon_{\mathrm{s}}}-1\right)\right]
\end{gathered}
$$

Further we have the form of the spectral shift in the vertical ionization of the charged particle,

$$
\Delta h v_{\mathrm{ab}}=\frac{1}{2 a}\left(Q_{1}-Q_{2}\right)^{2} \frac{\left(\varepsilon_{\mathrm{s}}-\varepsilon_{\mathrm{op}}\right)^{2}}{\left(\varepsilon_{\mathrm{s}}-1\right) \varepsilon_{\mathrm{op}}^{2} \varepsilon_{\mathrm{s}}}+\frac{1}{2 a}\left(Q_{1}^{2}-Q_{2}^{2}\right)\left(1-\frac{1}{\varepsilon_{\mathrm{s}}}\right)
$$

\subsubsection{Spectral shift of point dipole in a sphere cavity}

Here we will adopt Onsager model of sphere cavity and point dipole moment to treat the nonequilibrium polarization in spectrum. The solute charge distribution is considered as the point dipole, locating at the center of single vacuum sphere with the radius $a$, as shown in Figure 3. The solute cavity is surrounded by the solvent with dielectric constant $\varepsilon_{\mathrm{s}}$. The solute dipole will change from $\boldsymbol{\mu}_{1}$ to $\boldsymbol{\mu}_{2}$ due to the Franck-Condon transition in the light absorption process, and the light emission will lead to the inversed change of the solute dipole.

First, the reaction field in the sphere cavity will be derived. In Figure 2, the total electric potential $\Phi_{1}^{\mathrm{eq}}$ in equilibrium ground state satisfies the following differential equations and boundary conditions: 


$$
\begin{aligned}
& \left\{\begin{array}{l}
\nabla^{2} \varphi_{1, \text { in }}^{\mathrm{eq}}=0 \quad(r<a) ; \quad \nabla^{2} \Phi_{1, \text { out }}^{\mathrm{eq}}=0 \quad(r>a) \\
\Phi_{1, \text { in }}^{\mathrm{eq}}=\varphi_{1, \text { in }}^{\mathrm{eq}}+\frac{\mu_{1}}{r^{2}} \cos \theta
\end{array}\right. \\
& \left\{\begin{array}{l}
\left.\varphi_{1, \text { in }}^{\mathrm{eq}}\right|_{r \rightarrow 0} \text { is } \quad \text { finite, }\left.\quad \Phi_{1, \text { out }}^{\mathrm{eq}}\right|_{r \rightarrow \infty}=0 \\
\Phi_{1, \text { In }}^{\mathrm{eq}}=\Phi_{1, \text { out }}^{\mathrm{eq}}, \quad \frac{\partial \Phi_{1, \text { in }}^{\mathrm{eq}}}{\partial r}=\varepsilon_{s} \frac{\partial \Phi_{1, \text { out }}^{\mathrm{eq}}}{\partial r} \quad(r=a)
\end{array}\right.
\end{aligned}
$$

where the subscripts "in" and "out" stand for inside and outside the sphere cavity and " $\theta$ " is the angle between the vectors of solute dipole and $\mathbf{r}$. We assume that $\Phi_{1}^{\text {eq }}$ has the following form:

$$
\left\{\begin{array}{l}
\Phi_{1, \text { in }}^{\mathrm{eq}}=-E_{1 \mathrm{p}} r \cos \theta+\frac{\mu_{1}}{r^{2}} \cos \theta \\
\Phi_{1, \mathrm{out}}^{\mathrm{eq}}=\frac{\mu_{1}+\mu_{1 \mathrm{p}}}{r^{2}} \cos \theta
\end{array}\right.
$$

where the unknown $E_{1 \mathrm{p}}$ and $\mu_{1 \mathrm{p}}$ are polarization field strength and equivalent dipole for the solvent polarization. The above eq. (4.64) can satisfy the differential equation (4.62). So, substituting eq. (4.64) into (4.63) leads to

$$
\left\{\begin{array}{l}
-E_{1 \mathrm{p}} a+\frac{\mu_{1}}{a^{2}}=\frac{\mu_{1}+\mu_{1 \mathrm{p}}}{a^{2}} \\
-E_{1 \mathrm{p}}-\frac{2 \mu_{1}}{a^{3}}=-\varepsilon_{\mathrm{s}} \frac{2\left(\mu_{1}+\mu_{1 \mathrm{p}}\right)}{a^{3}}
\end{array}\right.
$$

then we can obtain

$$
E_{1 \mathrm{p}}=\frac{2\left(\varepsilon_{\mathrm{s}}-1\right)}{\left(2 \varepsilon_{\mathrm{s}}+1\right)} \frac{\mu_{1}}{a^{3}}, \mu_{1 \mathrm{p}}=-\frac{2\left(\varepsilon_{\mathrm{s}}-1\right)}{2 \varepsilon_{\mathrm{s}}+1} \mu_{1}
$$

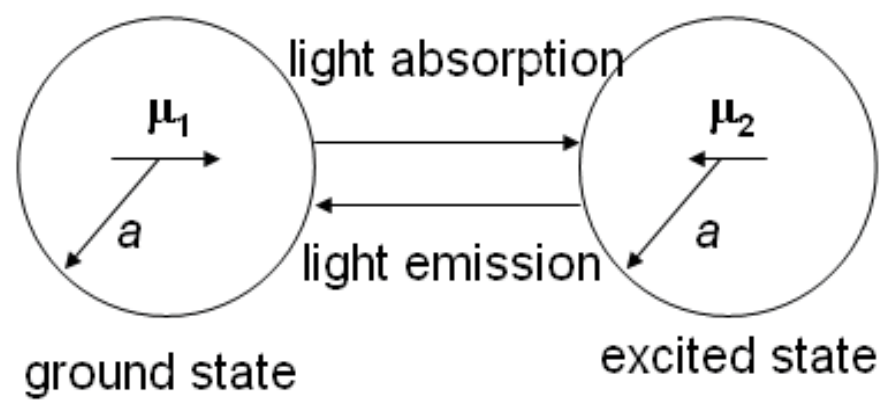

Fig. 3. Solvation model for single sphere 
By substituting eq. (4.66) into eq. (4.64), the potentials inside and outside the sphere are

$$
\left\{\begin{array}{l}
\Phi_{1, \text { in }}^{\mathrm{eq}}=-\frac{2\left(\varepsilon_{\mathrm{s}}-1\right)}{\left(2 \varepsilon_{\mathrm{s}}+1\right)} \frac{\mu_{1} r}{a^{3}} \cos \theta+\frac{\mu_{1}}{r^{2}} \cos \theta \\
\Phi_{1, \mathrm{out}}^{\mathrm{eq}}=\frac{3}{\left(2 \varepsilon_{\mathrm{s}}+1\right)} \frac{\mu_{1}}{r^{2}} \cos \theta
\end{array}\right.
$$

Therefore, the polarization potential inside the sphere cavity is

$$
\varphi_{1}=-\frac{2\left(\varepsilon_{\mathrm{s}}-1\right)}{\left(2 \varepsilon_{\mathrm{s}}+1\right)} \frac{\mu_{1}}{a^{3}} r \cos \theta
$$

Correspondingly, we can obtain the polarization field strength $\mathbf{E}_{1 \mathrm{p}}$ inside the sphere and the total field outside the sphere for equilibrium ground state as

$$
\begin{aligned}
\mathbf{E}_{1 \mathrm{p}} & =\frac{2\left(\varepsilon_{\mathrm{s}}-1\right)}{\left(2 \varepsilon_{\mathrm{s}}+1\right)} \frac{\boldsymbol{\mu}_{1}}{a^{3}} \\
\mathbf{E}_{1, \mathrm{out}} & =\frac{3}{\left(2 \varepsilon_{\mathrm{s}}+1\right)} \frac{\boldsymbol{\mu}_{1} \cdot \mathbf{r}}{r^{3}}
\end{aligned}
$$

Thus, the polarization field due to the dipole change $\Delta \boldsymbol{\mu}=\boldsymbol{\mu}_{2}-\boldsymbol{\mu}_{1}$ in medium with $\varepsilon_{\mathrm{s}}$ and $\varepsilon_{\text {op }}$ can be achieved as

$$
\Delta \mathbf{E}_{\mathrm{p}, \mathrm{eq}}=\frac{2\left(\varepsilon_{\mathrm{s}}-1\right)}{\left(2 \varepsilon_{\mathrm{s}}+1\right)} \frac{\Delta \boldsymbol{\mu}}{a^{3}}, \Delta \mathbf{E}_{\mathrm{p}, \mathrm{dy}}=\frac{2\left(\varepsilon_{\mathrm{op}}-1\right)}{\left(2 \varepsilon_{\mathrm{op}}+1\right)} \frac{\Delta \boldsymbol{\mu}}{a^{3}}
$$

Recalling eq. (C5), the introduced external dipole moment is

$$
\boldsymbol{\mu}_{\mathrm{ex}}=\frac{\varepsilon_{\mathrm{op}}-\varepsilon_{\mathrm{s}}}{\varepsilon_{\mathrm{s}}-1} \frac{3}{2 \varepsilon_{\mathrm{op}}+1} \Delta \boldsymbol{\mu}
$$

According to the definition of the solvent reorganization energy in eq. (4.34), we can obtain

$$
\begin{aligned}
\lambda_{\mathrm{s}} & =\frac{1}{2} \frac{\varepsilon_{\mathrm{op}}-\varepsilon_{\mathrm{s}}}{\varepsilon_{\mathrm{s}}-1} \frac{3}{2 \varepsilon_{\mathrm{op}}+1} \Delta \mathbf{\mu} \cdot\left[\frac{2\left(\varepsilon_{\mathrm{op}}-1\right)}{\left(2 \varepsilon_{\mathrm{op}}+1\right)} \frac{\Delta \boldsymbol{\mu}}{a^{3}}-\frac{2\left(\varepsilon_{\mathrm{s}}-1\right)}{\left(2 \varepsilon_{\mathrm{s}}+1\right)} \frac{\Delta \mathbf{\mu}}{a^{3}}\right] \\
& =\frac{(\Delta \mu)^{2}}{a^{3}} \frac{9\left(\varepsilon_{\mathrm{s}}-\varepsilon_{\mathrm{op}}\right)^{2}}{\left(\varepsilon_{\mathrm{s}}-1\right)\left(2 \varepsilon_{\mathrm{s}}+1\right)\left(2 \varepsilon_{\mathrm{op}}+1\right)^{2}}
\end{aligned}
$$

According to the definition in eq. (4.41), we obtain the final form for the absorption spectral shift with single sphere and point dipole approximation as 


$$
\begin{aligned}
& \Delta h v_{\mathrm{ab}}=\lambda_{\mathrm{s}}+\frac{1}{2}\left(\boldsymbol{\mu}_{1} \cdot \mathbf{E}_{1 \mathrm{p}}^{\mathrm{eq}}-\mathbf{\mu}_{2} \cdot \mathbf{E}_{2 \mathrm{p}}^{\mathrm{eq}}\right) \\
& =\frac{(\Delta \mu)^{2}}{a^{3}} \frac{9\left(\varepsilon_{\mathrm{s}}-\varepsilon_{\mathrm{op}}\right)^{2}}{\left(\varepsilon_{\mathrm{s}}-1\right)\left(2 \varepsilon_{\mathrm{s}}+1\right)\left(2 \varepsilon_{\mathrm{op}}+1\right)^{2}}+\frac{\left(\varepsilon_{\mathrm{s}}-1\right)}{\left(2 \varepsilon_{\mathrm{s}}+1\right)} \frac{\mu_{1}^{2}-\mu_{2}^{2}}{a^{3}}
\end{aligned}
$$

The similar treatment can lead to the emission spectral shift as

$$
\begin{aligned}
& \Delta h v_{\mathrm{em}}=\lambda_{\mathrm{s}}-\frac{1}{2}\left(\boldsymbol{\mu}_{1} \cdot \mathbf{E}_{1}^{\mathrm{eq}}-\boldsymbol{\mu}_{2} \cdot \mathbf{E}_{2}^{\mathrm{eq}}\right) \\
& =\frac{(\Delta \mu)^{2}}{a^{3}} \frac{9\left(\varepsilon_{\mathrm{s}}-\varepsilon_{\mathrm{op}}\right)^{2}}{\left(\varepsilon_{\mathrm{s}}-1\right)\left(2 \varepsilon_{\mathrm{s}}+1\right)\left(2 \varepsilon_{\mathrm{op}}+1\right)^{2}}-\frac{\left(\varepsilon_{\mathrm{s}}-1\right)}{\left(2 \varepsilon_{\mathrm{s}}+1\right)} \frac{\mu_{1}^{2}-\mu_{2}^{2}}{a^{3}}
\end{aligned}
$$

\subsection{Comments on traditional nonequilibrium solvation theory}

Nowadays, accompanying the development of computational methods and the progresses in computer science, solvent effect calculations at different levels have attracted much attention. Because most of chemistry and biochemistry reactions occur in solution, incorporation of the solvent effects into chemical models has been of great interest for several decades. Owing to the competitive advantages, continuum models are still playing a key role so far, although more and more explicit solvent methods, which take the microscopic structures of the solvent molecules into account, have been explored. There are two principal advantages of the continuum models. The first one is the reduction of the system's numbers of freedom degrees. If we take explicitly a few of solvent layers which involve hundreds of solvent molecules, a huge number of degrees of freedom will be added. The first thing we must face with is a large number of conformations. In addition, the observable structural and dynamical properties of some specific solute we most concern will be averaged. In fact, if one realizes that the complementary methods based on some explicit solvent methods are also not perfectly accurate, one will find the continuum model accounts for the dominant parts of solvent effects. So the second advantage of the macroscopic continuum models provides rather good ways to treat the strong and long-range electrostatic forces that dominate many solvation phenomena.

There are many circumstances in molecular modeling studies where a simplified description of solvent effects has advantages over the explicit modeling of each solvent molecule. The solute charge distribution and its response to the reaction field of the solvent dielectric, can be modeled either by quantum mechanics or by partial atomic charges in a molecular mechanics description. In spite of the severity of approximation of continuum models, it often gives a good account of equilibrium solvation energy, and hence widely used to estimate pKs, redox potential, and the electrostatic contributions to molecular solvation energy.

Up to now, several models for the equilibrium solvation based on the continuous medium theory were developed. The simplest one is the Onsager model with a point-dipole of solute in a spherical cavity. One of the most remarkable successes of the calculation of equilibrium solvation for arbitrary solute cavity is the establishment of polarizable continuum model (PCM) by Tomasi. Thereafter, different procedures for solute-solvent 
system have been developed. Introducing the numerical solution of the appropriate electrostatic potential into the popular quantum chemical packages yields different equilibrium solvation models. At present, a feature common to all the continuum solvation approximations is that the solute-solvent interactions are described in terms of the solute-reaction field interactions. The reaction field is due to the solvent polarization perturbed by the presence of solute, and the reaction field in turn perturbs the solute, until self-consistence is achieved. The reaction field is usually computed by solving the suitable Poisson equations.

So far, most of continuum models are properly referred to as equilibrium solvation models. Besides the structures and properties of a thermodynamically equilibrated solute-solvent system, the processes that take place on longer timescales may thus be legitimately thought of as equilibrium processes with respect to solvation. However, the question arises how to apply continuum models to the very fast processes. For instance, the transition state structures in principle live for only a single vibrational period. In such cases, the solvent response may not have time to equilibrate with the electronic state change at the position of transition state. Hence, a continuum model developed based on the fully equilibrated solvation would overestimate the solvation free energy by the assumed equilibration. In fact, many cases concern the nonequilibrium solvation problems in solution. The typical examples are: condensed-phase electron transfer, spectral shifts of photon absorption and emission in solution, and vibrational spectrum in solution. Among them, the solvent reorganization energy of the electron transfer and the spectral shifts attracted the most attention. So, in the present comments, we confine ourselves to these two kinds of nonequilibrium solvent effects, although the nonequilibrium solvation problem exists in some other processes such as proton transfer.

Let us date back to the beginning of the establishment of the nonequilibrium solvation theories. A brief overview on this topic will be helpful for us to clarify what fundamental defects exist in the present theories and application models. The concept of nonequilibrium solvation led to great progresses for people to understand the physics of fast processes in solution. Based on the separation of the two kinds of polarizations, orientational and electronic, Marcus applied the reversible work method to the establishment of the electrostatic free energy expression of the nonequilibrium solvation state. In Marcus' original treatments, the electrostatic free energy of nonequilibrium state of solution was defined as the sum of reversible works done during charging process involving two steps as follows ${ }^{[2]}$

$$
[\rho=0, \Phi=0] \stackrel{\mathrm{A} 1, \varepsilon_{\mathrm{S}}}{\longrightarrow}\left[\rho_{1}, \Phi_{1}^{\mathrm{eq}}\right] \stackrel{\mathrm{A} 2, \varepsilon_{\mathrm{op}}}{\longrightarrow}\left[\rho_{2}, \Phi_{2}^{\mathrm{non}}\right]
$$

In eq.(4.76), $\Phi$ denotes the total electric potential, including both the potential $\psi$ by the solute charge in vacuum and potential $\varphi$ due to the polarization of the medium. We confine our discussions only to the solute charge and the bound charge at present. The solute charge, which refers to the "free charge" from the viewpoint of electrodynamics, in principle represents the charge that can move about through the material. In practice what this ordinarily means is that free charges are not associated with any particular nucleus, but roam around at will. By contrast, the bound charges in dielectrics are attached to specific atoms or molecules. They are on a tight leash, and what they can do is to move a bit within 
the atom or molecule. Such microscopic displacements are not as dramatic as the wholesale rearrangement of solute charge, but their cumulative effects account for the characteristic behaviors of dielectric materials. For convenience, we call hereafter $\psi$ the vacuum potential and $\varphi$ the polarization potential. We do not distinguish "free charge" and "solute charge". Two terms, bound charge and polarized charge, are undistinguished in our previous works, but we use "bound charge" here.

In the establishment of the nonequilibrium state, the first step, A1, charges the solute to $\rho_{1}$, and $\Phi$ reaches an equilibrium in solvent of a static dielectric constant $\varepsilon_{\mathrm{s}}$. In step A2, the solute is charged from $\rho_{1}$ to $\rho_{2}$ but only the electronic component of the solvent polarization, which corresponds to the optical dielectric constant $\varepsilon_{\text {op }}$ of the solvent, responds. The system arrives at a new state in which the electronic polarization of solvent reaches equilibrium with $\rho_{2}$ but the orientaional polarization does not. This state, we denote it by $\left[\rho_{2}, \Phi_{2}^{\text {non }}\right]$, is referred to as the "nonequilibrium" state. If we note that the potential change in step A2 is caused by the change of solute charge, but only the electronic polarization responds, we can take the nonequilibrium as a superposition of two "equilibrium" states, $\left[\rho_{1}, \Phi_{1}^{\text {eq }}\right]$ and $\left[\Delta \rho, \Delta \Phi_{\mathrm{op}}\right]$. The former is a state in which $\rho_{1}$ equilibrates with the medium of dielectric constant of $\varepsilon_{\mathrm{s}}$, but the latter is such that the solute charge difference $\Delta \rho$ equilibrates in the hypothetical medium of a dielectric constant $\varepsilon_{\text {op }}$. Here we define the solute charge change and the potential change as

$$
\begin{aligned}
& \Delta \rho=\rho_{2}-\rho_{1} \\
& \Delta \Phi_{\text {op }}=\Phi_{2}^{\text {non }}-\Phi_{1}^{\text {eq }}
\end{aligned}
$$

As mentioned above, we divide the total potential $\Phi$ into two constitutive parts: $\psi$ due to the solute charge in vacuum and $\varphi$ due to the bound charge. We need to distinguish $\varphi^{\mathrm{eq}}$ of equilibrium from $\varphi^{\text {non }}$ of nonequilibrium for the polarization potential but this is unnecessary for $\psi$. Therefore we have

$$
\Phi_{i}^{\mathrm{eq}}=\psi_{i}+\varphi_{i}^{\mathrm{eq}}, \Phi_{i}^{\mathrm{non}}=\psi_{i}+\varphi_{i}^{\mathrm{non}} \quad(i=1,2)
$$

If we consider the inverse process of eq.(4.76) (denoted as as process B), we can write the analogue as

$$
[\rho=0, \Phi=0] \stackrel{\mathrm{B} 1, \varepsilon_{\mathrm{S}}}{\longrightarrow}\left[\rho_{2}, \Phi_{2}^{\mathrm{eq}}\right] \stackrel{\mathrm{B} 2, \varepsilon_{\mathrm{op}}}{\longrightarrow}\left[\rho_{1}, \Phi_{1}^{\mathrm{non}}\right]
$$

As mentioned above, we ignore the influence of solvent polarization upon the solute free charge, hence the charge distributions $\rho_{1}$ and $\rho_{2}$ in eq.(4.79) are supposed to be exactly the same as given in eq.(4.76).

If the properties of the dielectric do not vary during the process, it is very common to integrate the work done in the charging process by the following equation,

$$
\delta W=\int_{V} \Phi \delta \rho \mathrm{d} V
$$


The integration is over the whole space. Throughout this review, we use $W$ to denote the work done and $G$ the total free energy. But if we ignore the penetration of $\rho$ into the medium region, the integration will be in fact only carried out within the cavity occupied by the solute. Introducing a charging fraction $\alpha$ during step A1 of eq.(4.76), the electrostatic free energy of equilibrium state $\left[\rho_{1}, \Phi_{1}^{\mathrm{eq}}\right]$ was expressed in the well-known form, i.e.,

$$
\mathrm{G}_{1}^{\mathrm{eq}}=W_{\mathrm{A} 1}=(1 / 2) \int_{V} \rho_{1} \Phi_{1}^{\mathrm{eq}} \mathrm{d} V
$$

On the basis of step A1, step A2 introduces the further charge distribution change $\Delta \rho$, and the potential accordingly responds, so the charge distribution $\rho^{\alpha}$ and the total electric potential $\Phi^{\alpha}$ during step A2 were expressed by Marcus as ${ }^{[2]}$

$$
\rho^{\alpha}=\rho_{1}+\alpha\left(\rho_{2}-\rho_{1}\right) \quad \text { and } \Phi^{\alpha}=\Phi_{1}^{\mathrm{eq}}+\alpha\left(\Phi_{2}^{\mathrm{non}}-\Phi_{1}^{\mathrm{eq}}\right) \quad(\alpha=0 \sim 1)
$$

Therefore, the electrostatic free energy of nonequilibrium state was expressed by Marcus as the sum of work done in steps A1 and A2 [eq.(17) of ref.2], i.e.

$$
G_{2}^{\text {non }}(\mathrm{A})=(1 / 2) \int_{V}\left(\rho_{2} \Phi_{2}^{\text {non }}+\rho_{2} \Phi_{1}^{\mathrm{eq}}-\rho_{1} \Phi_{2}^{\mathrm{non}}\right) \mathrm{d} V
$$

Our following arguments will make it clear that eq.(4.83) is incorrect owing to the different response properties of the medium in equilibrium and nonequilibrium cases.

In the work of Marcus, the solvent reorganization energy is defined as the difference of electrostatic free energies between the nonequilibrium state and the equilibrium state subject to the same solute charge distribution, i.e.,

$$
\lambda_{\mathrm{o}}=G_{2}^{\mathrm{non}}-G_{2}^{\mathrm{eq}}
$$

Introducing the two-sphere approximation, the famous two-sphere model (as given by eq.(4.52) of estimating the reorganization energy was consequently developed and widely applied for decades. However, the Marcus two-sphere model often overestimates the solvent reorganization energy was, by a factor of about two for many electron transfer reactions ${ }^{[10,11]}$. For example, Basilevsky[12] developed a numerical method to evaluate the reorganization energy and applied it to the well-known Closs-Miller ET systems by using the conventional Marcus theory. However, the calculated values for the biphenyl-bridgenaphthalene system were exaggerated by a factor of about 2 than those fitted from the experimental rate constants.

The classical issue on the electrostatic free energy of nonequilibrium solvation in a continuous medium is revisited. The central idea, which has never been considered before, is to introduce a constrained equilibrium that is required to have the same charge distribution, polarization and entropy as the true nonequilibrium state (see Sections 1 3). Such a reference is certainly realizable via a quasistatic procedure. The location of the source for the tuning electric field $\mathbf{E}_{\mathrm{ex}}$ is yet completely irrelevant. From this reference, the electrostatic free energy of nonequilibrium solvation can directly be obtained in strict accordance with the principle of thermodynamics. It is also shown that the long lasting 
problem that the solvent reorganization energy is always overestimated by the previous continuum models is solved in a natural manner. It is believed that the present paradigm is completely general and can be used to derive other thermodynamic quantities of the isothermal nonequilibrium system as well.

The freezing of the state variables here is quite different from the treatment by Marcus. In fact, to freeze the variables of any nonequilibrium state is not only an abstract idea, but also a proper arrangement which can be used to realize the freezing. The fundamental difference between the Marcus approach and the present strategy is obvious. The freezing of the inertial polarization in Marcus work is just an idea without any measure, while our work realizes the freezing by introducing an external field. In our work, the whole polarization is kept frozen, not only the inertial part.

We mention that here the problem in the traditional nonequilibrium solvation theory arises from the simple reversible work integration, without consideration of any variable that describes the nonequilibrium state. A reversible work method applying to a non-quasistatic process is obviously arbitrary and lack of thermodynamic support. On the contrary, in our treatment, we rigorously obey the thermodynamics and a crucial external variable $\mathbf{E}_{\mathrm{ex}}$, which is used to constrain the nonequilibrium state to an "equilibrium" one, enters the expression of solvent reorganization energy. More details can be found in the references $4 \sim 6$.

\section{Appendix}

5.1 Appendix A : Proof of $\int \mathbf{E}_{2 c} \cdot \mathbf{P}^{\prime} \mathrm{d} V=\int \mathbf{E}_{\mathrm{ex}} \cdot \mathbf{P}_{2}^{\mathrm{eq}} \mathrm{d} V$

In the constrained equilibrium state, there is the relations of $\mathbf{P}^{\prime}=\chi_{s} \mathbf{E}^{\prime}$ and $\mathbf{P}_{2}^{\mathrm{eq}}=\chi_{s} \mathbf{E}_{2}^{\mathrm{eq}}$, thus we have

$$
\begin{gathered}
\int_{V} \mathbf{E}_{2}^{\mathrm{eq}} \cdot \mathbf{P}^{\prime} \mathrm{d} V=\int_{V} \chi_{\mathrm{s}} \mathbf{E}_{2}^{\mathrm{eq}} \cdot \mathbf{E}^{\prime} \mathrm{d} V=\int_{V} \mathbf{E}^{\prime} \cdot \mathbf{P}_{2}^{\mathrm{eq}} \mathrm{d} V \\
\int_{V} \mathbf{E}_{2 \mathrm{c}} \cdot \mathbf{P}^{\prime} \mathrm{d} V+\int_{V} \mathbf{E}_{2 \mathrm{p}} \cdot \mathbf{P}^{\prime} \mathrm{d} V=\int_{V} \mathbf{E}_{\mathrm{ex}} \cdot \mathbf{P}_{2}^{\mathrm{eq}} \mathrm{d} V+\int_{V} \mathbf{E}_{\mathrm{p}^{\prime}} \cdot \mathbf{P}_{2}^{\mathrm{eq}} \mathrm{d} V
\end{gathered}
$$

Applying the formulas of $\nabla \cdot \mathbf{P}=0$, and $\mathbf{n} \cdot \mathbf{P}=\sigma$ with $\sigma$ being the surface polarized charge density, the second term on the left hand side of the above equation can be rewritten as

$$
\begin{aligned}
& \int_{V} \mathbf{E}_{2 \mathrm{p}} \cdot \mathbf{P}^{\prime} \mathrm{d} V=-\int_{V} \nabla \varphi_{2 \mathrm{p}} \cdot \mathbf{P}^{\prime} \mathrm{d} V=-\int_{V} \nabla \cdot\left(\varphi_{2 \mathrm{p}} \mathbf{P}^{\prime}\right) \mathrm{d} V \\
& =-\int_{S} \varphi_{2 \mathrm{p}} \mathbf{n} \cdot \mathbf{P}^{\prime} \mathrm{d} S=-\int_{S} \varphi_{2 \mathrm{p}}(\mathbf{r}) \sigma^{\prime}(\mathbf{r}) \mathrm{d} S=-\int_{S} \int_{S^{\prime}} \sigma^{\prime}(\mathbf{r}) \frac{\sigma_{2 \mathrm{p}}\left(\mathbf{r}^{\prime}\right)}{\left|\mathbf{r}-\mathbf{r}^{\prime}\right|} \mathrm{d} S \mathrm{~d} S^{\prime}
\end{aligned}
$$

where $\sigma_{2 p}$ and $\sigma^{\prime}$ represent the polarization surface charges corresponding to $\mathbf{E}_{2 p}$ and $\mathbf{P}^{\prime}$ respectively, while $\varphi_{2 p}$ denotes the equilibrium polarization potential, $\mathbf{E}_{2 p}=-\nabla \varphi_{2 p}$. In the same way, the second term on the right hand side of eq. (A2) can be changed to the following form, 


$$
\int_{V} \mathbf{E}_{\mathrm{p}}^{\prime} \cdot \mathbf{P}_{2}^{\mathrm{eq}} \mathrm{d} V=-\int_{V} \nabla \varphi^{\prime} \cdot \mathbf{P}_{2}^{\mathrm{eq}} \mathrm{d} V=-\int_{S} \int_{S^{\prime}} \sigma^{\prime}(\mathbf{r}) \frac{\sigma_{2 \mathrm{p}}\left(\mathbf{r}^{\prime}\right)}{\left|\mathbf{r}-\mathbf{r}^{\prime}\right|} \mathrm{d} S \mathrm{~d} S^{\prime}
$$

Substituting eqs. (A3) and (A4) into eq. (A2), we obtain the desired equality

$$
\int \mathbf{E}_{2 \mathrm{c}} \cdot \mathbf{P}^{\prime} \mathrm{d} V=\int \mathbf{E}_{\mathrm{ex}} \cdot \mathbf{P}_{2}^{\mathrm{eq}} \mathrm{d} V
$$

\subsection{Appendix B: The proof for the solvent reorganization energy in charge-potential} form

In the equilibrium medium, the divergency of the solvent polarization and surface polarized charge $\sigma$ can be expressed as

$$
\nabla \cdot \mathbf{P}=0, \mathbf{n} \cdot \mathbf{P}=\sigma
$$

Further by using $\Delta \mathbf{E}_{\mathrm{dy}}=-\nabla\left(\Delta \Phi_{\mathrm{dy}}\right)$, we have

$$
\begin{aligned}
\lambda_{\mathrm{s}} & \left.=\frac{1}{2} \frac{\varepsilon_{\mathrm{s}}-\varepsilon_{\mathrm{op}}}{\varepsilon_{\mathrm{s}}-1} \int_{V} \nabla\left(\Delta \Phi_{\mathrm{dy}}\right) \cdot\left(\Delta \mathbf{P}_{\mathrm{dy}}-\Delta \mathbf{P}_{\mathrm{eq}}\right)\right] \mathrm{d} V \\
& =\frac{1}{2} \frac{\varepsilon_{\mathrm{s}}-\varepsilon_{\mathrm{op}}}{\varepsilon_{\mathrm{s}}-1} \int_{V} \nabla \cdot\left[\Delta \Phi_{\mathrm{dy}}\left(\Delta \mathbf{P}_{\mathrm{dy}}-\Delta \mathbf{P}_{\mathrm{eq}}\right)\right] \mathrm{d} V \\
& =\frac{1}{2} \frac{\varepsilon_{\mathrm{s}}-\varepsilon_{\mathrm{op}}}{\varepsilon_{\mathrm{s}}-1} \oint_{S} \Delta \Phi_{\mathrm{dy}}\left(\Delta \mathbf{P}_{\mathrm{dy}}-\Delta \mathbf{P}_{\mathrm{eq}}\right) \cdot \mathbf{n d} S
\end{aligned}
$$

that is,

$$
\lambda_{\mathrm{s}}=\frac{1}{2} \frac{\varepsilon_{\mathrm{s}}-\varepsilon_{\mathrm{op}}}{\varepsilon_{\mathrm{s}}-1} \oint_{S} \Delta \Phi_{\mathrm{dy}}\left(\Delta \sigma_{\mathrm{dy}}-\Delta \sigma_{\mathrm{eq}}\right) \mathrm{d} S
$$

which is applicable to solute cavities of general shapes and sizes.

\subsection{Appendix C : The brief expression for the solvent reorganization energy in sphere cavity model}

If the point charge $q$ of the solute locate at the center of the sphere cavity with the radius of $r$, it will produce the electric field strength in vacuum as

$$
\mathbf{E}_{\mathrm{c}}=\frac{q \mathbf{r}}{r^{2}}
$$

Then we can set a point charge $q_{\mathrm{ex}}$ at the center of the solute sphere, defined as 


$$
q_{\mathrm{ex}}=\frac{\varepsilon_{\mathrm{op}}-\varepsilon_{\mathrm{s}}}{\varepsilon_{\mathrm{s}}-1} \frac{1}{\varepsilon_{\mathrm{op}}} \Delta q
$$

with $\Delta q=q_{2}-q_{1}$, it can generate the needed external field strength in vacuum as

$$
\mathbf{E}_{\mathrm{ex}}=\frac{\varepsilon_{\mathrm{op}}-\varepsilon_{\mathrm{s}}}{\varepsilon_{\mathrm{s}}-1} \frac{\Delta q \mathbf{r}}{\varepsilon_{\mathrm{op}} r^{2}}=\left(\frac{\chi_{\mathrm{op}}}{\chi_{\mathrm{s}}}-1\right) \Delta \mathbf{E}_{\mathrm{dy}}
$$

If the solute charge can be regarded as the point dipole $\boldsymbol{\mu}$ at the sphere center, the field strength produce by it in vacuum is

$$
\mathbf{E}_{\mathrm{c}}=\frac{\boldsymbol{\mu} \cdot \mathbf{r}}{r^{3}}
$$

If we can place another point dipole $\boldsymbol{\mu}_{\mathrm{ex}}$ at the center, defined by

$$
\boldsymbol{\mu}_{\mathrm{ex}}=\frac{\varepsilon_{\mathrm{op}}-\varepsilon_{\mathrm{s}}}{\left(\varepsilon_{\mathrm{s}}-1\right)} \frac{3}{2 \varepsilon_{\mathrm{op}}+1} \Delta \boldsymbol{\mu}
$$

then there will be the needed external field strength $\mathbf{E}_{\mathrm{ex}}$ as

$$
\mathbf{E}_{\mathrm{ex}}=\frac{\varepsilon_{\mathrm{op}}-\varepsilon_{\mathrm{s}}}{\left(\varepsilon_{\mathrm{s}}-1\right)} \frac{3}{2 \varepsilon_{\mathrm{op}}+1} \frac{\Delta \boldsymbol{\mu} \cdot \mathbf{r}}{r^{3}}=\left(\frac{\chi_{\mathrm{op}}}{\chi_{\mathrm{s}}}-1\right) \Delta \mathbf{E}_{\mathrm{dy}}
$$

by using the relation of $\Delta \mathbf{E}_{\mathrm{dy}}=\frac{3}{2 \varepsilon_{\mathrm{op}}+1} \frac{\Delta \boldsymbol{\mu} \cdot \mathbf{r}}{r^{3}}$. It should be noticed that $\mathbf{E}_{\mathrm{ex}}$ is the vacuum field strength due to external charge and it will generate the additional polarization $\mathbf{P}^{\prime}$, polarization field $\mathbf{E}_{\mathrm{p}}{ }^{\prime}$ and polarization potential $\varphi^{\prime}$ as

$$
\mathbf{P}^{\prime}=\Delta \mathbf{P}_{\mathrm{dy}}-\Delta \mathbf{P}_{\mathrm{eq}}, \mathbf{E}_{\mathrm{p}}{ }^{\prime}=\Delta \mathbf{E}_{\mathrm{dy}}-\Delta \mathbf{E}_{\mathrm{eq}}, \varphi^{\prime}=\Delta \varphi_{\mathrm{dy}}-\Delta \varphi_{\mathrm{eq}}
$$

in the medium with dielectric constant $\varepsilon_{s}$. By using $\mathbf{E}_{\mathrm{ex}}=-\nabla \psi_{\mathrm{ex}}$ with $\psi_{\mathrm{ex}}$ being the vacuum potential due to $\mathbf{P}^{\prime}$, we can obtain

$$
\begin{aligned}
& \lambda_{\mathrm{s}}=\frac{1}{2} \int \mathbf{E}_{\mathrm{ex}} \cdot \mathbf{P}^{\prime} \mathrm{d} V=-\frac{1}{2} \int \mathbf{P}^{\prime} \cdot \nabla \psi_{\mathrm{ex}} \mathrm{d} V=\frac{1}{2} \int \psi_{\mathrm{ex}} \nabla \cdot \mathbf{P}^{\prime} \mathrm{d} V=-\frac{1}{2} \int \psi_{\mathrm{ex}}(\mathbf{r}) \rho^{\prime}(\mathbf{r}) \mathrm{d} V \\
& =-\frac{1}{2} \int_{V V^{\prime}} \frac{\rho_{\mathrm{ex}}\left(\mathbf{r}^{\prime}\right) d V^{\prime}}{\left|\mathbf{r}-\mathbf{r}^{\prime}\right|} \rho^{\prime}(\mathbf{r}) \mathrm{d} V=-\frac{1}{2} \iint_{V^{\prime}} \frac{\rho^{\prime}(\mathbf{r}) d V}{\left|\mathbf{r}-\mathbf{r}^{\prime}\right|} \rho_{\mathrm{ex}}\left(\mathbf{r}^{\prime}\right) \mathrm{d} V^{\prime}=-\frac{1}{2} \int \rho_{\mathrm{ex}} \varphi^{\prime} \mathrm{d} V
\end{aligned}
$$


where $\rho^{\prime}$ is the polarized charge due to $\mathbf{E}_{\mathrm{ex}}$ in the medium. Substituting eq. (C7) into the above equation, it can be obtained that

$$
\lambda_{\mathrm{s}}=\frac{1}{2} \int \rho_{\mathrm{ex}}\left(\Delta \varphi_{\mathrm{eq}}-\Delta \varphi_{\mathrm{dy}}\right) \mathrm{d} V
$$

This equation is the brief expression for the solvent reorganization energy with sphere cavity approximation.

In the case of solute charge being point charge, eq. (C9) can be simplified as

$$
\lambda_{\mathrm{s}}=\frac{1}{2} q_{\mathrm{ex}}\left(\Delta \varphi_{\mathrm{eq}}-\Delta \varphi_{\mathrm{dy}}\right)
$$

In another case with point charges $q_{\mathrm{D}}$ and $q_{\mathrm{A}}$ locating at the centers of electron donor's and acceptor's spheres, eq. (C9) can be rewritten as

$$
\lambda_{\mathrm{s}}=\frac{1}{2} q_{\mathrm{D}, \mathrm{ex}}\left(\Delta \varphi_{\mathrm{D}, \mathrm{eq}}-\Delta \varphi_{\mathrm{D}, \mathrm{dy}}\right)+\frac{1}{2} q_{\mathrm{A}, \mathrm{ex}}\left(\Delta \varphi_{\mathrm{A}, \mathrm{eq}}-\Delta \varphi_{\mathrm{A}, \mathrm{dy}}\right)
$$

In the case of solute point dipole, the dipole can be expressed as the product of the charge $q$ and distance $d \mathbf{l}$, i.e., $\boldsymbol{\mu}=q d \mathbf{l}$, thus we have

$$
q \varphi_{+}-q \varphi_{-}=q \mathrm{~d} \varphi=q d \mathbf{l} \cdot \nabla \varphi=-\mathbf{\mu} \cdot \mathbf{E}
$$

According to eqs. (C9) and (C12), the solvent reorganization energy with point dipole and sphere cavity approximation can be expressed as

$$
\lambda_{\mathrm{s}}=\frac{1}{2} \boldsymbol{\mu}_{\mathrm{ex}} \cdot\left(\Delta \mathbf{E}_{\mathrm{p}, \mathrm{dy}}-\Delta \mathbf{E}_{\mathrm{p}, \mathrm{eq}}\right)
$$

\section{References}

[1] (a) Leontovich M. A. An Introduction to Thermodynamics, 2nd ed, Gittl Publ, Moscow, 1950 (in Russian). (b) Leontovich M. A. Introduction to Thermodynamics, Statistical Physics 2nd; Nauka: Moscow, 1983( in Russian).

[2] Marcus R. A. J. Chem. Phys. 1956, 24: 979.

[3] Pekar S. I. Introduction into Electronic Theory of Crystals, Technical Literature Publishers, Moscow, 1951.

[4] Li X.-Y., He F.-C., Fu K.-X., Liu W. J. Theor. Comput. Chem. 2010, 9(supp.1): 23. 
[5] Wang X.-J., Zhu Q., Li Y.-K., Cheng X.-M., Fu K.-X., Li X.-Y. J. Phys. Chem. B. 2010, 114: 2189.

[6] Li X.-Y., Wang Q.-D., Wang J.-B., Ma J.-Y., Fu K.-X., He F.-C. Phys. Chem. Chem. Phys. 2010, 12: 1341.

[7] Jackson J. D. Classical Electrodynamics, Third Edition, John Wiley \& Sons, Inc. New York, 1999: 165-168.

[8] Landau L. D., Lifshitz E. M., Pitaevskii L. P. Eletronynamics of Continous Media, 2nd ed. Butterworth-Heinemman, Ltd, 1984.

[9] Marcus R. A. J. Phys. Chem. 1994, 98: 7170.

[10] Johnson M. D., Miller J. R., Green N. S., Closs G. L. J. Phys. Chem. 1989, 93: 1173.

[11] Formasinho S. J., Arnaut L. G., Fausto R. Prog. Reaction. Kinetics. 1998, $23: 1$.

[12] Basilevsky M. V., Chudinov G. E., Rostov I. V., Liu Y., Newton M. D. J. Mol. Struct. Theochem. 1996, 371: 191. 


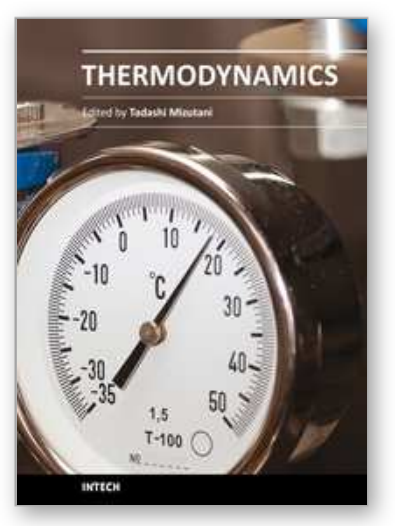

\author{
Thermodynamics \\ Edited by Prof. Mizutani Tadashi
}

ISBN 978-953-307-544-0

Hard cover, 440 pages

Publisher InTech

Published online 14, January, 2011

Published in print edition January, 2011

Progress of thermodynamics has been stimulated by the findings of a variety of fields of science and technology. The principles of thermodynamics are so general that the application is widespread to such fields as solid state physics, chemistry, biology, astronomical science, materials science, and chemical engineering. The contents of this book should be of help to many scientists and engineers.

\title{
How to reference
}

In order to correctly reference this scholarly work, feel free to copy and paste the following:

Li Xiang-Yuan, Zhu Quan, He Fu-Cheng and Fu Ke-Xiang (2011). Extension of Classical Thermodynamics to Nonequilibrium Polarization, Thermodynamics, Prof. Mizutani Tadashi (Ed.), ISBN: 978-953-307-544-0, InTech, Available from: http://www.intechopen.com/books/thermodynamics/extension-of-classicalthermodynamics-to-nonequilibrium-polarization

\section{INTECH}

open science | open minds

\author{
InTech Europe \\ University Campus STeP Ri \\ Slavka Krautzeka 83/A \\ 51000 Rijeka, Croatia \\ Phone: +385 (51) 770447 \\ Fax: +385 (51) 686166 \\ www.intechopen.com
}

\author{
InTech China \\ Unit 405, Office Block, Hotel Equatorial Shanghai \\ No.65, Yan An Road (West), Shanghai, 200040, China \\ 中国上海市延安西路65号上海国际贵都大饭店办公楼 405 单元 \\ Phone: +86-21-62489820 \\ Fax: $+86-21-62489821$
}


(C) 2011 The Author(s). Licensee IntechOpen. This chapter is distributed under the terms of the Creative Commons Attribution-NonCommercialShareAlike-3.0 License, which permits use, distribution and reproduction for non-commercial purposes, provided the original is properly cited and derivative works building on this content are distributed under the same license. 